\title{
Overview of Meta-Analyses: The Impact of Dietary Lifestyle on Stroke Risk
}

\author{
Emma Altobelli ${ }^{1, *}$, Paolo Matteo Angeletti ${ }^{1}$, Leonardo Rapacchietta ${ }^{1}$ and Reimondo Petrocelli ${ }^{2}$ \\ 1 Department of Life, Health and Environmental Sciences, Epidemiology and Biostatistics Unit, \\ University of L'Aquila, 67100 L'Aquila, Italy; paolomatteoangeletti@gmail.com (P.M.A.); \\ leonardo.rapacchietta@gmail.com (L.R.) \\ 2 Public Health Unit, ASREM, 86100, Campobasso, Italy; reimondo.petrocelli@asrem.org \\ * Correspondence: emma.altobelli@cc.univaq.it; Tel.: +39-0862-434666
}

Received: 12 August 2019; Accepted: 20 September 2019; Published: 25 September 2019

check for updates

\begin{abstract}
A stroke is one of the most prevalent cardiovascular diseases worldwide, both in high-income countries and in medium and low-medium income countries. The World Health Organization's (WHO) report on non-communicable diseases (NCDs) indicates that the highest behavioral risk in NCDs is attributable to incorrect nutrition. The objective of our work is to present an overview of meta-analyses that have investigated the impact of different foods and/or drinks in relationship with the risk of stroke events (ischemic/hemorrhagic). The papers to be included in the overview were found in MEDLINE, EMBASE, Scopus, Clinicaltrials.gov, Web of Science, and Cochrane Library and were selected according to the preferred reporting items for systematic reviews and meta-analyses (PRISMA) flow chart. Quality assessment were made according to the AMSTAR 2 scale. This overview shows that all primary studies came from countries with high income levels. This evidence shows that many countries are not represented. Therefore, different lifestyles, ethnic groups, potentially harmful or virtuous eating habits are not reported. It is important to underline how the choose of foods may help reduce the risk of cardiovascular diseases and stroke in particular.
\end{abstract}

Keywords: overview; meta-analyses; stroke; nutrition; geographical areas

\section{Introduction}

A stroke is one of the most prevalent cardiovascular diseases worldwide. It is estimated that in 2010 there were $11,569,538$ ischemic stroke events, $63 \%$ of which were in medium and low-medium income countries [1]. In the same year, 5,324,997 hemorrhagic strokes occurred, $80 \%$ of which were in medium and low-medium income areas [1]. This difference is similar for mortality, which is significantly lower in high-income countries compared to those of middle/medium-low [1]. It is estimated that in Europe the costs of the disease are around $€ 7775$ per patient, with a total cost, in billions, of 64,053 euros [2]. In the United States in 2008 the global costs were estimated to be 62.5 billion dollars, the expenditure forecast for 2050 is about 2.2 trillion dollars [3].

The World Health Organization's (WHO) report on non-communicable diseases (NCDs) indicates that the highest behavioral risk is attributable to incorrect nutrition, particularly in the WHO European region [4].

Numerous meta-analysis studies have been conducted to evaluate the relationship between diet and stroke risk. A meta-analysis by Alexander et al. [5] seems to indicate a protective action resulting from the consumption of cheese. This data is in line with Briggs et al. [6]. Dairy products should probably be consumed as part of a balanced diet in which there is adequate intake of all nutrients within an appropriate calorie count [7-11]. 
Regarding alcohol use and/or abuse [12], red wine contains polyphenols, including resveratrol, a molecule with not only cardio protective pleiotropic effects, but also neuroprotective, anti-microbial and anti-angiogenetic effects [13]. All this has a positive influence on the prevention of ischemic stroke since it acts on one of the main causes, atrial fibrillation [14]. The same may be extended to moderate consumption of beer [15].

Monounsaturated [16] and polyunsaturated [17] fats have been considered as a valid nutritional support: monounsaturated fatty acids (MUFAs) seems to positively modify lipid structure in patients [18].

In addition, it is important to underline consumption of fruit and vegetables [19]. The Centers for Disease Control and Prevention guidelines recommend the daily consumption of 1.5-2.0 cups of fruit and 2.0-3.0 cups of vegetables [20].

Consumption of nuts could have a protective role in decreasing the risk of cardiovascular disease $[21,22]$. The benefit of the intake of nuts seems to be linked to the composition of polyunsaturated fatty acids (PUFAs) that improve the performance of the cardiovascular system as reported by Del Gobbo et al. [23].

Finally, the increased risk of ischemic stroke in women who consume high quantities of sugary drinks is due to the insulin peak resulting in the ingestion of large amounts of glucose [24]. Furthermore, it is important to take into consideration how many of these drinks contain added fructose. Fructose enters the glycolytic pathway downregulating it with the intermediate products of its metabolism (glycerol 3-phosphate and acetyl CoA), thus favoring lipogenesis and accumulation of intramuscular and visceral fat [25].

Tea [26] seems to have a role in stroke prevention, as reported by Arab and colleagues as well as folic acid [27-29]. The consumption of whole grains does not present significant results [30].

The objective of our work is to present an overview of meta-analyses that have investigated the impact of different foods and/or drinks in relationship with the risk of stroke events (ischemic/hemorrhagic). We considered the meta-analyses based on cohort studies and randomized clinical trials.

\section{Materials and Methods}

Meta-analyses regarding the onset of hemorrhagic and/or ischemic strokes in subjects following dietary regimes with a given food or specific nutritional or nutraceutical support have been considered. In addition, studies investigating secondary prevention of strokes were considered, also in relation to a specific food or nutritional or nutraceutical support.

The papers included in the overview were sought in the last 10 years in MEDLINE, EMBASE, Scopus, Clinicaltrials.gov, Web of Science, and Cochrane Library databases up until 31 December 2018. The search strategy was conducted using the following terms: Stroke OR Strokes OR Cerebrovascular Accident OR CVAs (Cerebrovascular Accidents) OR Cerebrovascular Apoplexy, Cerebrovascular OR Vascular Accident, Brain OR Brain Vascular Accident OR Brain Vascular Accidents OR Vascular Accidents OR Brain OR Cerebrovascular Stroke OR Cerebrovascular Strokes OR Stroke, Cerebrovascular OR Strokes, Cerebrovascular OR Apoplexy OR Cerebral Stroke OR Cerebral Strokes OR Stroke, Cerebral OR Strokes, Cerebral OR Stroke, Acute OR Acute Stroke OR Acute Strokes OR Strokes, Acute OR Cerebrovascular Accident Acute OR Acute Cerebrovascular Accident OR Acute Cerebrovascular Accidents OR Cerebrovascular Accidents, Acute AND "Food" (Mesh)) AND "Meta-Analysis" (publication type). The selection of works was conducted using the preferred reporting items for systematic reviews and meta-analyses (PRISMA) method [31] by two-blinded authors (P.M.A. and L.R.). A methodologist (E.A.) resolved any disagreements. To avoid redundant results, we only considered the last published meta-analysis for the same topic.

The quality of the meta-analyses was assessed using the AMSTAR 2 scale by Shea et al. [32] that evaluates the methodological quality of the meta-analyses (Supplementary Table S1). In addition, we evaluated the distribution of primary studies included in each meta-analysis according to six 
different geographical areas (Australia; Canada; China, Singapore and South Korea; Europe; Japan; and USA) and according to four nutritional patterns and/or product types (eating habits, food, beverage, nutrients).

\section{Results}

The methodology used is described in the preferred reporting items for systematic reviews and meta-analyses (PRISMA) flow chart (Figure 1).


Figure 1. The preferred reporting items for systematic reviews and meta-analyses (PRISMA) flow chart.

The literature search highlighted 189 references (Figure 1). After the exclusion of 131 references, the remaining 58 were analyzed by reading the full text, then 23 were excluded: three did not present the outcome of interest and the other 20 were excluded because they were less recent in respect to those included in the review that presented the same outcomes. In total, 35 articles were selected, of which 27 were meta-analyses based on observational studies and eight were randomized controlled trials (RCTs). Table 1 shows the studies by author and by food considered with the respective dose effects found. Table S2 shows the studies by author with the dose response analysis.

Table 2 and Figure 2 show the distribution of primary studies included in each meta-analysis, according to six different geographical areas (Australia; Canada; China, Singapore and South Korea; Europe; Japan; and USA) and according to four nutritional patterns and/or product types (eating habits, food, beverage, nutrients). 




Figure 2. Distribution of primary studies included in meta-analyses considered according to geographic area and type of nutritional support.

Graphical summary results of meta-analyses are reported in Figures 3-6.

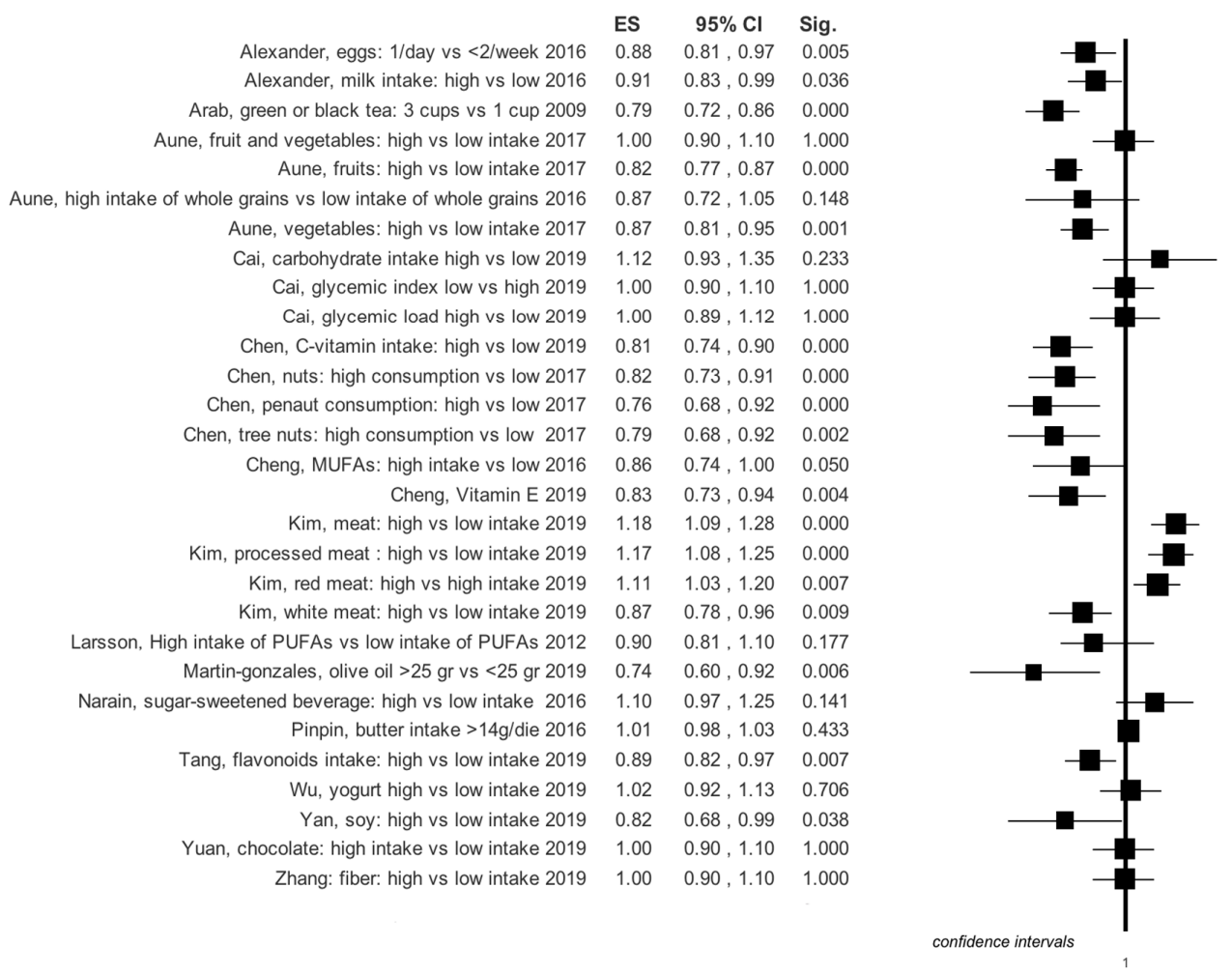

Figure 3. Summary results of effects size for any type of stroke events in observational studies, based on study design of selected primary studies for each meta-analysis. 


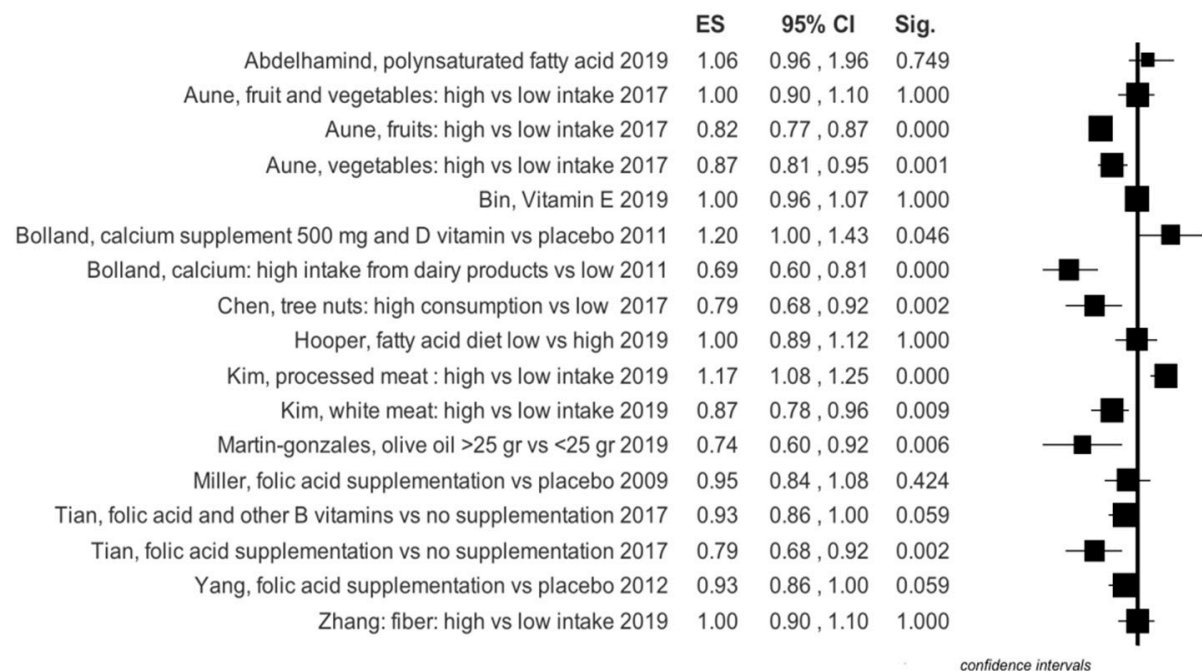

Figure 4. Summary results of effects size for any type of stroke events in RCT, based on study design of selected primary studies for each meta-analysis.

\begin{tabular}{|c|c|c|c|}
\hline Observational & ES & $95 \% \mathrm{Cl}$ & Sig. \\
\hline Alexander, milk intake: high vs low 2016 & 0.93 & $0.81,1.06$ & 0.290 \\
\hline Aune, high tree nuts consumption vs low tree nuts consumption 2016 & 0.93 & $0.77,1.13$ & 0.458 \\
\hline Chen, C-vitamin intake: high vs low 2019 & 0.77 & $0.64,0.92$ & 0.005 \\
\hline Cheng, MUFAs: high intake vs low 2016 & 0.92 & $0.79,1.08$ & 0.296 \\
\hline Larrson, Heavy drinkers vs no drinkers 2016 & 1.13 & $0.95,1.19$ & 0.033 \\
\hline Larrson, light-moderate drinkers vs never drinkers 2016 & 0.87 & $0.82,0.91$ & 0.000 \\
\hline Larsson, High intake of PUFAs vs low intake of PUFAs 2012 & 0.82 & $0.71,0.94$ & 0.006 \\
\hline Larsson, occasional drinkers vs light-moderate drinking 2016 & 0.98 & $0.94,1.04$ & 0.433 \\
\hline Narain, sugar-sweetened beverage: high vs low intake 2016 & 1.01 & $0.74,1.37$ & 0.950 \\
\hline \multicolumn{4}{|l|}{ RCT } \\
\hline Abdelhamid, alpha linoleic acid 2019 & 1.20 & $0.85,1.69$ & 0.298 \\
\hline Abdelhamid, long chain omega-3 2019 & 1.15 & $0.66,2.01$ & 0.623 \\
\hline Bin, Vitamin E 2019 & 1.01 & $0.94,1.09$ & 0.792 \\
\hline
\end{tabular}

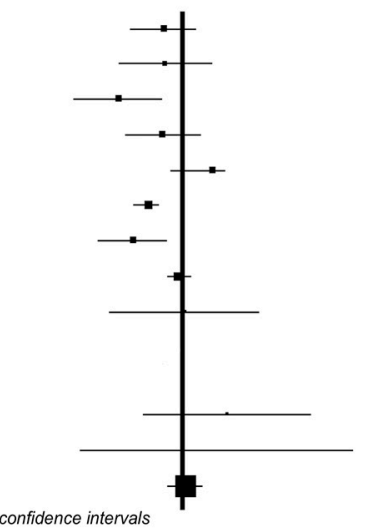

Figure 5. Summary results of effects size for ischemic of stroke events, based on study design of selected primary studies for each meta-analysis.

$\begin{array}{rlcl}\text { Observational } & \text { ES } & 95 \% \text { Cl } & \text { Sig. } \\ \text { Alexander, cheese: high vs low intake 2016 } & 0.87 & 0.77,0.99 & 0.030 \\ \text { Alexander, milk intake: high vs low 2016 } & 0.93 & 0.69,1.25 & 0.632 \\ \text { Chen, C-vitamin intake: high vs low 2019 } & 1.07 & 0.38,3.00 & 0.898 \\ \text { Cheng, MUFAs: high intake vs low 2016 } & 0.68 & 0.49,0.96 & 0.025 \\ \text { take of PUFAs vs low intake of PUFAs 2012 } & 0.80 & 0.55,1.15 & 0.236 \\ \text { weetened beverage: high vs low intake 2016 } & 0.87 & 0.68,1.12 & 0.274\end{array}$

\section{RCT}

Abdelhamid, alpha linoleic acid 2019

Abdelhamid, long chain omega-3 2019

$1.09 \quad 0.89,1.33$

$1.06 \quad 0.96,1.96$

0.400

Bin, Vitamin E 2019

1.12

$0.94,1.33$

0.749

0.201



Figure 6. Summary results of effects size for hemorrhagic of stroke events, based on study design of selected primary studies for each meta-analysis. 


\subsection{Dairy Products}

Four meta-analyses specifically investigated the use of milk and dairy products. In the work of Mullie et al. [8] it is evident that the consumption of $200 \mathrm{~mL}$ of milk does not lead to an increased risk of stroke, while Alexander et al. [5] show that risk reduction appears to border statistical significance. Surprisingly, however, the consumption of cheese seems to reduce stroke risk (Table 1, Figure 3). The latter author has also performed a dose-response analysis which suggests that in total the intake of dairy products is protective against stroke; specifically, the daily consumption of cheese with a range from $0.5-1.5$ servings; in particular, an intake of calcium from dairy products of $100-300 \mathrm{mg} / \mathrm{dL}$ or above $300 \mathrm{mg} / \mathrm{dL}$ also helps to protect (Table S2). On the other hand, a single meta-analysis investigated the correlation between risk of developing stroke and consumption of butter [9] and did not show a statistically significant increase in risk (Table 1, Figure 3). A paper by Wu et al. was concerned specifically with yogurt consumption, but its outcome was not statistically significant, risk reduction $(R R)=1.02(0.92-1.13)$ [33]. This evidence was similar also in the dose-response analysis for quantities below $200 \mathrm{~g} /$ day, $R R=1.06$ (0.98-1.15) and for quantities above $200 \mathrm{~g} / \mathrm{day}, \mathrm{RR}=0.92(0.85-1.00)$ [31] . Instead, the more controversial use of calcium along with vitamin $D$ vs. a placebo shows an $R R=1.20$ (1.00-1.43) (Table 1, Figure 3) [34].

\subsection{Alcohol Consumption}

Two meta-analyses have been identified that identify alcohol as a risk factor for stroke [7,12]. It is possible to summarize the effect of alcohol on stroke substantially as a biphasic effect: protective, if consumed within the limits of 1-2 alcoholic units but very detrimental in the case of more than 4 alcoholic units (conventionally, a drink containing $8 \mathrm{mg}$ of ethanol is identified as an alcoholic unit). Specifically, the consumption of alcohol seems to be protective in ischemic stroke when comparing mild and moderate consumption vs. non-drinkers, with an $R R=0.87(0.81-0.92)$ (Table 1, Figure 5). As for the impact of alcohol on hemorrhagic stroke, heavy drinkers show a markedly higher risk for the onset of an intracerebral hemorrhage when compared to the occasional drinker, $R R=1.74$ (1.45-2.09) (Table 1, Figure 6) [12]. Larsson et al. [12] performed a dose-effect analysis to confirm the above data. The consumption of 1-2 alcoholic units a day has a protective effect against ischemic stroke. On the other hand, consumption of 4 alcoholic units is associated with an increased risk of ischemic or hemorrhagic stroke (Table S2) [12].

Zhang's meta-analysis also shows how a moderate consumption of alcohol has a protective effect compared to heavy consumption (Table 1, Figure 3) [7].

\subsection{Monounsaturated Fatty Acids (MUFAs) and Polyunsaturated Fatty Acids (PUFAs)}

A meta-analysis with 10 cohort studies included [16] investigated the consumption of MUFAs; its results show that RR is at the limits of statistical significance (Table 1, Figure 4).

Meta-analyses of Abdelhamid [35] and Hooper [36] on RCTs showed that omega-3 and omega-6 do not influence stroke risk, respectively: $R R=1.06(0.96-1.16)$ and $R R=1.36(0.45-4.11)$. Larsson et al. [17] investigated the consumption of PUFAs, also on cohort studies, finding these molecules to be protective of ischemic stroke (Table 1, Figure 5). On the contrary, Abdelhaimid's meta-analysis [37] on RCTs showed a non-significant PUFA effect on stroke risk: $R R=0.91(0.58-1.44)$.

\subsection{Saturated Fatty Acids}

Muto et al. [38] investigated the effect of a diet rich in saturated fatty acids. They showed that with regard to ischemic stroke, the overall RR was 0.89 (0.82-0.96), while it was 0.68 (0.47-0.96) for hemorrhagic stroke. Not significant results were found in Hooper et al. meta-analysis (Table 1, Figures 5 and 6) [39]. 


\subsection{Olive Oil}

Martin-Gonzales' meta-analysis has highlighted that olive oil consumption has a protective effect against stroke: $R R=0.74(0.60-0.92)$ (Table 1, Figure 3) [40].

\subsection{Vitamin $E$}

The results of a meta-analysis by Cheng et al. regarding observational studies, highlighted that vitamin E supplements decrease stroke risk: $R R=0.83$ (0.73-0.94) (Table 1, Figure 3) [41]. On the other hand, a meta-analysis on RCTs by Bin et al. [42] showed that vitamin E supplements are irrelevant to stroke onset: $R R=1.01(0.94-1.07)$.

\subsection{Hazelnuts}

Chen [21] investigated the consumption of nuts and the incidence of stroke. The consumption of hazelnuts appears to be protective against stroke (Table 1, Figure 3). There are, however, some differences regarding the consumption of different types of hazelnuts (Table 1, Figure 3).

In the dose-effect study, Chen showed how a weekly consumption of up to five portions could reduce mortality [21] (Table S2).

\subsection{Black and Green Tea}

A meta-analysis by Arab et al. [26] investigated the consumption of green and black tea as a protective factor against the onset of stroke. The results, shown in Table 1, appear to be rather encouraging, favoring a reduction in the risk of stroke (Table 1, Figure 3).

\subsection{Sugary Drinks}

Narain et al. [43] studied the consumption of sugary drinks, determining how a high intake of such drinks, especially in women, seems to favor ischemic stroke (Table 1, Figure 5).

\subsection{Whole Grains}

One meta-analysis investigated the protective use of whole grains in the development of cardiovascular diseases and also strokes [30]. This evidence was confirmed even after the dose-response analysis (Table S2).

\subsection{Fruit and Vegetables}

Aune's research illustrated the benefit of consumption of fruits and vegetables against the onset of stroke (Table 1, Figure 3). The benefit appears evident in the dose-response study, particularly for certain categories of plant-based foods, such as citrus fruits and citrus juices, for ischemic and hemorrhagic stroke, and the consumption of leafy vegetables for the onset of only ischemic stroke [19] (Table S2).

\subsection{Vitamin B Complex}

A recent meta-analysis shows that folic acid can reduce stroke risk with an $\mathrm{RR}=0.79(0.68-0.92)$; while, the combined intake of folic acid and other B-complex vitamins does not appear to be significant, with an RR = $0.91(0.82-1.00)$ (Table 1, Figure 3) [27].

\subsection{Carbohydrate Intake}

A meta-analysis analyzed the incidence of stroke with respect to the total consumption of carbohydrates as well as glycemic index and glycemic load [44]. The risk of stroke incidence was significant in foods with a higher glycemic load: $R R=1.19(1.05-1.36)$. No statistical significance was 
found for the consumption of the glycemic carbohydrate index $(R R=1.1,0.99-1.21)$ and for global carbohydrate consumption ( $R R=1.12,0.93-1.25$ ) (Table 1, Figure 3) [44].

\subsection{Soy}

A meta-analysis investigated soy consumption and analyzed 11 observational studies, including four case-controls and seven cohort studies [45]. The categories with high soy consumption were compared to those with low soy consumption. In the cumulative analysis soy consumption reduced the risk of stroke significantly $(R R=0.82,0.68-0.99)$ (Table 1, Figure 3) [45].

\subsection{Fibers}

A meta-analysis by Zhang et al. on fiber consumption highlighted how high fiber intakes are associated with a stroke reduction. In particular, high fiber consumption proved to be protective in ischemic stroke ( $R R=0.83,0.74-0.93)$, but not in hemorrhagic stroke $(R R=0.87,0.72-1.05)$. The dose-response analysis showed that the daily intake of $5 \mathrm{~g}$ of fiber leads to a risk reduction $(R R=0.90$, 0.82-0.99). A further increase of $10 \mathrm{~g}$ shows a higher decrease of $R R=0.84(0.75-0.94)$ (Table 1 , Figure 3) [46].

\subsection{Protein}

Zhang et al. [47] showed that total protein consumption does not affect stroke risk. However, the consumption of vegetable proteins could be protective ( $R R=0.90 ; 0.82 ; 0.99)$ (Table 1 , Figure 3$)$.

\subsection{Fish}

Qin's meta-analysis investigated fish consumption [48]. There is no significant relative risk in the comparison between the consumption of lean fish and fatty fish $(R R=0.88 ; 0.74-1.04)$, while there is a protective effect in the consumption of large quantities of lean fish compared to the consumption of small quantities of lean fish ( $R R=0.81 ; 0.67-0.99)$. Xun's meta-analysis [49] showed how large consumption of fish has a protective effect against stroke: $R R=0.91(0.85-0.98)$ (Table 1, Figure 3).

\subsection{Meat}

Kim et al. investigated the incidence of stroke with respect to meat consumption. Red meat consumption was associated with an increased risk $(R R=1.11 ; 1.03-1.20)$. On the other hand, there was a protective effect in the consumption of white meat $(R R=0.87 ; 0.78-0.96)$ (Table 1 , Figure 3) [50].

\subsection{Chocolate}

Chocolate consumption shows a protective effect against stroke: $R R=0.84(0.78-0.90)$ (Table 1 , Figure 3) [51].

\subsection{Flavonoids}

High consumption of flavonoids investigated in the meta-analysis by Tang et al. is stroke protective $(R R=0.89 ; 0.82-0.97)$. A daily increase of $100 \mathrm{~g}$ showed no statistically significant results $(R R=0.91$; 0.77-1.08) (Table 1, Figure 3) [52].

\subsection{Vitamin $C$}

The meta-analysis of Chen et al. concerned vitamin $C$ intake [53]. Consumption of high doses was preventive in the development of ischemic or hemorrhagic stroke ( $R R=0.81 ; 0.74-0.90)$. Similarly, the dose-response analysis verified that the incremental intake of $100 \mathrm{mg} /$ day of vitamin $\mathrm{C}$ has a protective role in the incidence of stroke, $R R=0.82(0.75-0.93)$ (Table S2). In particular, the intake of vitamin $C$ would seem to be protective against ischemic stroke, $R R=0.77$ (0.64-0.92), but not hemorrhagic $(R R=1.07 ; 0.38-3.00)$ (Table 1, Figures 3-6). 


\subsection{Legumes}

The consumption of $100 \mathrm{~g}$ per week of pulses showed $\mathrm{RR}=1.07(0.77-1.50)$, with regard to ischemic stroke and $R R=1.23(0.91 ; 1.66)$ as regards to hemorrhagic stroke (Table 1, Figure 3) [54].

\subsection{Eggs}

A moderate consumption of eggs is associated with a potential decrease of stroke, $R R=0.88$ (0.81-0.97) (Table 1, Figure 3) [55].

\subsection{Geographical Distribution of Primary Studies}

As regards to geographical distribution of the primary studies, with respect to beverage, food, eating habits or nutrients, there is a strong difference among the areas considered (Figure 2, Table 2). Europe and the USA are areas where the majority of studies were conducted: 162 in Europe (42\%) and 130 in the USA (33.7\%). It is important to underline that studies about diet style were not conducted in Canada and Australia.

Eight studies on cereals were conduct in Europe (4.9\%), 5 in the USA (3.4\%), 3 in Japan (5\%), and 1 in China-Singapore-Korea (4\%). There was a similar trend for fruits and vegetables: 22 (13.6\%) studies in Europe, 14 in the USA (11.6\%), 7 in Japan (11.6\%), and 1 (4\%) in China-Korea-Singapore area.

It is important to underline that Japan followed Europe and the USA in studies pertaining to alcohol use (Figure 2, Table 2); respectively, they have conducted 7 (11.6\%), 10 (6.2\%), and $13(5.3 \%)$ works respectively, while only 2 studies were done in the China-Korea-Singapore region (9\%). All areas considered have studied nutrients (omega-3) with particular attention (Figure 2, Table 2). 
Table 1. Characteristics of included meta-analyses in the overview according to food or beverage, study design, and type of stroke.

\begin{tabular}{|c|c|c|c|c|c|c|c|c|c|}
\hline \multirow{2}{*}{ Author } & \multirow{2}{*}{ Food or Beverage } & \multirow{2}{*}{ Control Group } & \multirow{2}{*}{$\begin{array}{c}\text { Literature } \\
\text { Search Update }\end{array}$} & \multirow{2}{*}{$\begin{array}{c}\text { Number of } \\
\text { Primary Studies }\end{array}$} & \multirow{2}{*}{ Type of Strokes * } & \multirow{2}{*}{$\begin{array}{l}\text { Number of Studies for } \\
\text { Evaluated Strokes }\end{array}$} & \multicolumn{2}{|c|}{ Population } & \multirow{2}{*}{$\begin{array}{l}\text { Effect Size } \\
95 \% \text { CI }\end{array}$} \\
\hline & & & & & & & No. Total & No. Events & \\
\hline \multicolumn{10}{|c|}{ Observational Studies } \\
\hline \multirow{5}{*}{ Alexander [5] } & High milk intake & Low milk intake & \multirow{5}{*}{2016} & \multirow{5}{*}{$\mathrm{K}=31$} & $\begin{array}{l}\text { Ischemic or } \\
\text { Hemorrhagic }\end{array}$ & 7 & - & - & $0.91(0.83 ; 0.99)$ \\
\hline & High milk intake & Low milk intake & & & $\begin{array}{c}\text { Ischemic or } \\
\text { Hemorrhagic (in men) }\end{array}$ & 4 & - & - & $1.04(0.96 ; 1.14)$ \\
\hline & High milk intake & Low milk intake & & & $\begin{array}{l}\text { Ischemic or } \\
\text { Hemorrhagic }\end{array}$ & 4 & - & - & $0.93(0.81 ; 1.06)$ \\
\hline & High milk intake & Low milk intake & & & Hemorrhagic & 3 & - & - & $0.93(0.69 ; 1.25)$ \\
\hline & High cheese intake & Low cheese intake & & & $\begin{array}{l}\text { Ischemic or } \\
\text { Hemorrhagic }\end{array}$ & 4 & - & - & $0.87(0.77 ; 0.99)$ \\
\hline \multirow[b]{2}{*}{ Zhang [7] } & $\begin{array}{c}\text { Western dietary } \\
\text { pattern-high categories \# }\end{array}$ & $\begin{array}{c}\text { Western dietary } \\
\text { pattern-low categories \# }\end{array}$ & \multirow[b]{2}{*}{2015} & $\mathrm{~K}=21$ & $\begin{array}{l}\text { Ischemic or } \\
\text { Hemorrhagic }\end{array}$ & 8 & 143,798 & 2049 & $1.05(0.82 ; 1.35)^{* *}$ \\
\hline & $\begin{array}{c}\text { Healthy dietary } \\
\text { pattern-high categories \# }\end{array}$ & $\begin{array}{c}\text { Healthy dietary } \\
\text { pattern-low categories \# }\end{array}$ & & & & 14 & 318,813 & 3971 & $0.77(0.64 ; 0.93)^{* *}$ \\
\hline \multirow[b]{2}{*}{ Mullie [8] } & \multirow{2}{*}{$\begin{array}{l}200 \mathrm{~mL} / \text { day daily milk } \\
\text { consumption }\end{array}$} & \multirow[b]{2}{*}{ No milk consumption } & \multirow[t]{2}{*}{2016} & \multirow[t]{2}{*}{$\mathrm{K}=19$} & $\begin{array}{l}\text { Ischemic or } \\
\text { Hemorrhagic }\end{array}$ & 10 & 567,717 & 39,352 & $0.91(0.82 ; 1.02)$ \\
\hline & & & & & $\begin{array}{c}\text { Ischemic or } \\
\text { Hemorrhagic (in men) }\end{array}$ & 5 & - & - & $0.96(0.86 ; 1.09)$ \\
\hline Pimpin [9] & Butter intake $<14 \mathrm{~g} /$ day & Butter intake $>14$ g/day & 2016 & $\mathrm{~K}=4$ & $\begin{array}{l}\text { Ischemic or } \\
\text { Hemorrhagic }\end{array}$ & 3 & 173,853 & 5229 & $1.01(0.98 ; 1.03)$ \\
\hline \multirow{14}{*}{ Larsson [12] } & Light-moderate drinking & No drinkers & \multirow{14}{*}{2016} & \multirow{14}{*}{$\mathrm{K}=27$} & \multirow{6}{*}{ Ischemic stroke } & 8 & - & - & $0.87(0.81 ; 0.92)$ \\
\hline & Heavy drinking & No drinkers & & & & 8 & - & - & $1.13(0.95 ; 1.19)$ \\
\hline & Light-moderate drinking & Never drinkers & & & & 8 & - & - & $0.87(0.82 ; 0.91)$ \\
\hline & Heavy drinking & Never drinkers & & & & 8 & - & - & $1.06(0.95 ; 1.19)$ \\
\hline & Occasional drinking & Light-moderate drinkers & & & & 8 & - & - & $0.98(0.94 ; 1.04)$ \\
\hline & Heavy drinking & Occasional drinkers & & & & 8 & - & - & $1.13(1.03 ; 1.24)$ \\
\hline & Light-moderate drinking & No drinkers & & & \multirow{4}{*}{$\begin{array}{l}\text { Intracerebral } \\
\text { hemorrhage }\end{array}$} & 5 & - & - & $0.91(0.64 ; 1.29)$ \\
\hline & Heavy drinking & No drinkers & & & & 4 & - & - & $1.21(0.87 ; 1.67)$ \\
\hline & Light-moderate drinking & Occasional drinkers & & & & 4 & - & - & $1.04(0.89 ; 1.21)$ \\
\hline & Heavy drinking & Occasional drinkers & & & & 4 & - & - & $1.74(1.45 ; 2.09)$ \\
\hline & Light-moderate drinking & No drinkers & & & \multirow{4}{*}{$\begin{array}{l}\text { Subarachnoid } \\
\text { Hemorrhage events }\end{array}$} & 5 & - & - & $1.39(1.00 ; 1.92)$ \\
\hline & Heavy drinking & No drinkers & & & & 3 & - & - & $1.43(1.00 ; 2.05)$ \\
\hline & Light-moderate drinking & Occasional drinkers & & & & 4 & - & - & $1.10(0.84 ; 1.44)$ \\
\hline & Heavy drinking & Occasional drinkers & & & & 4 & - & - & $1.62(0.89 ; 2.29)$ \\
\hline
\end{tabular}


Table 1. Cont.

\begin{tabular}{|c|c|c|c|c|c|c|c|c|c|}
\hline \multirow{2}{*}{ Author } & \multirow{2}{*}{ Food or Beverage } & \multirow{2}{*}{ Control Group } & \multirow{2}{*}{$\begin{array}{l}\text { Literature } \\
\text { Search Update }\end{array}$} & \multirow{2}{*}{$\begin{array}{c}\text { Number of } \\
\text { Primary Studies }\end{array}$} & \multirow{2}{*}{ Type of Strokes * } & \multirow{2}{*}{$\begin{array}{l}\text { Number of Studies for } \\
\text { Evaluated Strokes }\end{array}$} & \multicolumn{2}{|c|}{ Population } & \multirow{2}{*}{$\begin{array}{l}\text { Effect Size } \\
95 \% \mathrm{CI}\end{array}$} \\
\hline & & & & & & & No. Total & No. Events & \\
\hline \multirow{3}{*}{ Cheng [16] } & \multirow{3}{*}{$\begin{array}{l}\text { High monounsaturated } \\
\text { fatty acids (MUFAs) intake }\end{array}$} & \multirow{3}{*}{ Low usage of MUFAs } & \multirow{3}{*}{2016} & \multirow{3}{*}{$\mathrm{K}=10$} & $\begin{array}{l}\text { Ischemic or } \\
\text { Hemorrhagic }\end{array}$ & 10 & 314,511 & 5827 & $0.86(0.74 ; 1.00)$ \\
\hline & & & & & Ischemic stroke & 8 & & - & $0.92(0.79 ; 1.08)$ \\
\hline & & & & & Hemorrhagic stroke & 5 & - & - & $0.68(0.49 ; 0.96)$ \\
\hline \multirow{3}{*}{ Larsson [17] } & \multirow{3}{*}{$\begin{array}{l}\text { High long-chain omega-3 } \\
\text { polyunsaturated fatty acids } \\
\text { (PUFAs) intake }\end{array}$} & \multirow{3}{*}{ Low intake of PUFA } & \multirow{3}{*}{2012} & \multirow{3}{*}{$\mathrm{K}=10$} & $\begin{array}{l}\text { Ischemic or } \\
\text { Hemorrhagic }\end{array}$ & 10 & 242,076 & 5238 & $0.90(0.81 ; 1.10)$ \\
\hline & & & & & Ischemic stroke & 5 & - & - & $0.82(0.71 ; 0.94)$ \\
\hline & & & & & Hemorrhagic stroke & 5 & - & - & $0.80(0.55 ; 1.15)$ \\
\hline $\begin{array}{c}\text { Martin- } \\
\text { Gonzales [40] }\end{array}$ & Olive oil (>25 g) & Olive oil $(<25 \mathrm{~g})$ & 2014 & $\mathrm{~K}=2$ & $\begin{array}{l}\text { Ischemic or } \\
\text { Hemorrhagic }\end{array}$ & 2 & - & - & $0.74(0.60 ; 0.92)$ \\
\hline Cheng [41] & Vitamin E & - & 2018 & $K=9$ & $\begin{array}{l}\text { Ischemic or } \\
\text { Hemorrhagic }\end{array}$ & 9 & - & - & $0.83(0.73 ; 0.94)$ \\
\hline \multirow{13}{*}{ Aune [19] } & $\begin{array}{l}\text { High intake of fruit and } \\
\text { vegetables }\end{array}$ & $\begin{array}{l}\text { Low intake of fruit and } \\
\text { vegetables }\end{array}$ & \multirow{13}{*}{2017} & \multirow{13}{*}{$\mathrm{K}=95$} & $\begin{array}{l}\text { Ischemic or } \\
\text { Hemorrhagic }\end{array}$ & 8 & 226,910 & 10,560 & $0.79(0.71 ; 0.88)$ \\
\hline & High intake of fruit & Low intake of fruit & & & $\begin{array}{l}\text { Ischemic or } \\
\text { Hemorrhagic }\end{array}$ & 17 & 960,337 & 46,951 & $0.82(0.77 ; 0.87)$ \\
\hline & High intake vegetables & Low intake vegetables & & & $\begin{array}{l}\text { Ischemic or } \\
\text { Hemorrhagic }\end{array}$ & 13 & 427,124 & 14,519 & $0.87(0.81 ; 0.95)$ \\
\hline & $\begin{array}{l}\text { High intake apples and } \\
\text { pears }\end{array}$ & $\begin{array}{l}\text { Low intake apples and } \\
\text { pears }\end{array}$ & & & $\begin{array}{l}\text { Ischemic or } \\
\text { Hemorrhagic }\end{array}$ & 6 & - & - & $0.88(0.81 ; 0.96)$ \\
\hline & High intake berries & Low intake berries & & & $\begin{array}{l}\text { Ischemic or } \\
\text { Hemorrhagic }\end{array}$ & 5 & - & - & $0.98(0.86 ; 1.12)$ \\
\hline & High intake citrus fruits & Low intake citrus fruits & & & $\begin{array}{l}\text { Ischemic or } \\
\text { Hemorrhagic }\end{array}$ & 8 & - & - & $0.74(0.65 ; 0.84)$ \\
\hline & $\begin{array}{l}\text { High intake citrus } \\
\text { fruit juice }\end{array}$ & $\begin{array}{l}\text { Low intake citrus } \\
\text { fruit juice }\end{array}$ & & & $\begin{array}{l}\text { Ischemic or } \\
\text { Hemorrhagic }\end{array}$ & 2 & - & - & $0.90(0.74 ; 1.10)$ \\
\hline & High intake dried fruits & Low intake dried fruits & & & $\begin{array}{l}\text { Ischemic or } \\
\text { Hemorrhagic }\end{array}$ & 2 & - & - & $0.92(0.74 ; 1.15)$ \\
\hline & High intake fruits juice & Low intake fruit juice & & & $\begin{array}{l}\text { Ischemic or } \\
\text { Hemorrhagic }\end{array}$ & 2 & - & - & $0.67(0.60 ; 0.76)$ \\
\hline & High intake grapes & Low intake grapes & & & $\begin{array}{l}\text { Ischemic or } \\
\text { Hemorrhagic }\end{array}$ & 2 & - & - & $0.72(0.47 ; 1.10)$ \\
\hline & $\begin{array}{l}\text { High intake allium } \\
\text { vegetables }\end{array}$ & $\begin{array}{l}\text { Low intake allium } \\
\text { vegetables }\end{array}$ & & & $\begin{array}{l}\text { Ischemic or } \\
\text { Hemorrhagic }\end{array}$ & 2 & - & - & $0.89(0.80 ; 1.00)$ \\
\hline & $\begin{array}{l}\text { High intake cruciferous } \\
\text { vegetables }\end{array}$ & $\begin{array}{l}\text { Low intake cruciferous } \\
\text { vegetables }\end{array}$ & & & $\begin{array}{l}\text { Ischemic or } \\
\text { Hemorrhagic }\end{array}$ & 4 & - & - & $0.97(0.78 ; 1.20)$ \\
\hline & $\begin{array}{l}\text { High intake green leafy } \\
\text { vegetables }\end{array}$ & $\begin{array}{l}\text { Low intake green leafy } \\
\text { vegetables }\end{array}$ & & & $\begin{array}{l}\text { Ischemic or } \\
\text { Hemorrhagic }\end{array}$ & 4 & - & - & $0.88(0.81 ; 0.95)$ \\
\hline
\end{tabular}


Table 1. Cont.

\begin{tabular}{|c|c|c|c|c|c|c|c|c|c|}
\hline \multirow{2}{*}{ Author } & \multirow{2}{*}{ Food or Beverage } & \multirow{2}{*}{ Control Group } & \multirow{2}{*}{$\begin{array}{c}\text { Literature } \\
\text { Search Update }\end{array}$} & \multirow{2}{*}{$\begin{array}{c}\text { Number of } \\
\text { Primary Studies }\end{array}$} & \multirow{2}{*}{ Type of Strokes * } & \multirow{2}{*}{$\begin{array}{l}\text { Number of Studies for } \\
\text { Evaluated Strokes }\end{array}$} & \multicolumn{2}{|c|}{ Population } & \multirow{2}{*}{$\begin{array}{l}\text { Effect Size } \\
95 \% \text { CI }\end{array}$} \\
\hline & & & & & & & No. Total & No. Events & \\
\hline \multirow{18}{*}{ Aune [19] } & $\begin{array}{l}\text { High intake pickled } \\
\text { vegetables }\end{array}$ & $\begin{array}{l}\text { Low intake pickled } \\
\text { vegetables }\end{array}$ & & & $\begin{array}{l}\text { Ischemic or } \\
\text { Hemorrhagic }\end{array}$ & 2 & - & - & $0.80(0.73 ; 0.88)$ \\
\hline & High intake potatoes & Low intake potatoes & & & $\begin{array}{l}\text { Ischemic or } \\
\text { Hemorrhagic }\end{array}$ & 4 & - & - & $0.94(0.87 ; 1.01)$ \\
\hline & High intake root vegetables & $\begin{array}{c}\text { Low intake root } \\
\text { vegetables }\end{array}$ & & & $\begin{array}{l}\text { Ischemic or } \\
\text { Hemorrhagic }\end{array}$ & 2 & - & - & $1.01(0.89 ; 1.14)$ \\
\hline & High intake tomatoes & Low intake tomatoes & & & $\begin{array}{l}\text { Ischemic or } \\
\text { Hemorrhagic }\end{array}$ & 3 & - & - & $0.95(0.68 ; 1.31)$ \\
\hline & High intake berries & Low intake berries & & & Ischemic & 3 & - & - & $0.95(0.75 ; 1.21)$ \\
\hline & High intake citrus fruits & Low intake citrus fruits & & & Ischemic & 7 & - & - & $0.78(0.66 ; 0.92)$ \\
\hline & $\begin{array}{l}\text { High intake citrus fruit } \\
\text { juice }\end{array}$ & $\begin{array}{l}\text { Low intake citrus fruit } \\
\text { juice }\end{array}$ & & & Ischemic & 2 & - & - & $0.65(0.51 ; 0.84)$ \\
\hline & $\begin{array}{c}\text { High intake allium } \\
\text { vegetables }\end{array}$ & $\begin{array}{l}\text { Low intake allium } \\
\text { vegetables }\end{array}$ & & & Ischemic & 2 & - & - & $0.90(0.78 ; 1.03)$ \\
\hline & $\begin{array}{l}\text { High intake cruciferous } \\
\text { vegetables }\end{array}$ & $\begin{array}{c}\text { Low intake cruciferous } \\
\text { vegetables }\end{array}$ & & & Ischemic & 5 & - & - & $0.82(0.66 ; 1.01)$ \\
\hline & $\begin{array}{c}\text { High intake green leafy } \\
\text { vegetables }\end{array}$ & $\begin{array}{c}\text { Low intake green leafy } \\
\text { vegetables }\end{array}$ & & & Ischemic & 4 & - & - & $0.88(0.78 ; 0.99)$ \\
\hline & High intake potatoes & Low intake potatoes & & & Ischemic & 5 & - & - & $0.97(0.87 ; 1.08)$ \\
\hline & High intake root vegetables & $\begin{array}{c}\text { Low intake root } \\
\text { vegetables }\end{array}$ & & & Ischemic & 3 & - & - & $0.93(0.73 ; 1.18)$ \\
\hline & High intake tomatoes & Low intake tomatoes & & & Ischemic & 2 & - & - & $0.80(0.69 ; 0.92)$ \\
\hline & High intake berries & Low intake berries & & & Hemorrhagic & 3 & - & - & $1.15(0.89 ; 1.49)$ \\
\hline & High intake citrus fruits & Low intake citrus fruits & & & Hemorrhagic & 3 & - & - & $0.74(0.55 ; 1.01)$ \\
\hline & $\begin{array}{l}\text { High intake cruciferous } \\
\text { vegetables }\end{array}$ & $\begin{array}{c}\text { Low intake cruciferous } \\
\text { vegetables }\end{array}$ & & & Hemorrhagic & 2 & - & - & $0.83(0.33 ; 2.12)$ \\
\hline & High intake potatoes & Low intake potatoes & & & Hemorrhagic stroke & 3 & - & - & $1.06(0.83 ; 1.36)$ \\
\hline & High intake root vegetables & $\begin{array}{c}\text { Low intake root } \\
\text { vegetables }\end{array}$ & & & Hemorrhagic stroke & 2 & - & - & $1.05(0.76 ; 1.44)$ \\
\hline \multirow{4}{*}{ Chen [21] } & All nuts high consumption & $\begin{array}{l}\text { All nuts low } \\
\text { consumption }\end{array}$ & \multirow{4}{*}{2017} & \multirow{4}{*}{$K=16$} & \multirow{4}{*}{$\begin{array}{l}\text { Ischemic or } \\
\text { Hemorrhagic }\end{array}$} & 12 & 449,293 & 4398 & $0.82(0.73 ; 0.91)$ \\
\hline & $\begin{array}{l}\text { Nut plus peanut butter } \\
\text { high consumption }\end{array}$ & $\begin{array}{l}\text { Nut plus peanut butter } \\
\text { low consumption }\end{array}$ & & & & 3 & 104,531 & 924 & $0.84(0.70 ; 1.01)$ \\
\hline & Peanuts high consumption & $\begin{array}{l}\text { Peanuts low } \\
\text { consumption }\end{array}$ & & & & 5 & 265,252 & 7025 & $0.76(0.69 ; 0.82)$ \\
\hline & $\begin{array}{l}\text { Tree nuts high } \\
\text { consumption }\end{array}$ & $\begin{array}{l}\text { Tree nuts low } \\
\text { consumption }\end{array}$ & & & & 3 & 130,987 & 6394 & $0.79(0.68 ; 0.92)$ \\
\hline
\end{tabular}


Table 1. Cont.

\begin{tabular}{|c|c|c|c|c|c|c|c|c|c|}
\hline \multirow{2}{*}{ Author } & \multirow{2}{*}{ Food or Beverage } & \multirow{2}{*}{ Control Group } & \multirow{2}{*}{$\begin{array}{c}\text { Literature } \\
\text { Search Update }\end{array}$} & \multirow{2}{*}{$\begin{array}{c}\text { Number of } \\
\text { Primary Studies }\end{array}$} & \multirow{2}{*}{ Type of Strokes * } & \multirow{2}{*}{$\begin{array}{l}\text { Number of Studies for } \\
\text { Evaluated Strokes }\end{array}$} & \multicolumn{2}{|c|}{ Population } & \multirow{2}{*}{$\begin{array}{l}\text { Effect Size } \\
95 \% \mathrm{CI}\end{array}$} \\
\hline & & & & & & & No. Total & No. Events & \\
\hline \multirow{5}{*}{ Aune [30] } & $\begin{array}{l}\text { High intake of whole } \\
\text { grains or specific types of } \\
\text { grains }\end{array}$ & $\begin{array}{l}\text { Low intake of whole } \\
\text { grains or specific types of } \\
\text { grains }\end{array}$ & \multirow{5}{*}{2016} & \multirow{5}{*}{$\mathrm{K}=15$} & \multirow{5}{*}{$\begin{array}{l}\text { Ischemic or } \\
\text { Hemorrhagic }\end{array}$} & 5 & - & - & $0.87(0.72 ; 1.05)$ \\
\hline & $\begin{array}{l}\text { High intake whole grain } \\
\text { bread }\end{array}$ & $\begin{array}{l}\text { Low intake whole grain } \\
\text { bread }\end{array}$ & & & & 2 & - & - & $0.88(0.75 ; 1.03)$ \\
\hline & $\begin{array}{c}\text { High intake of whole grain } \\
\text { breakfast cereals } \\
\end{array}$ & $\begin{array}{l}\text { Low intake of whole } \\
\text { grain breakfast cereals }\end{array}$ & & & & 2 & - & - & $0.99(0.53 ; 1.86)$ \\
\hline & High intake of refined grain & $\begin{array}{c}\text { Low intake of refined } \\
\text { grain }\end{array}$ & & & & 4 & - & - & $0.95(0.78 ; 1.14)$ \\
\hline & High intake total rice & Low intake total rice & & & & 4 & - & - & $1.02(0.94 ; 1.11)$ \\
\hline Wu [33] & High yogurt intake & Low yogurt intake & 2017 & $\mathrm{~K}=7$ & $\begin{array}{l}\text { Ischemic or } \\
\text { Hemorrhagic }\end{array}$ & 7 & - & - & $1.02(0.92 ; 1.13)$ \\
\hline Muto [38] & $\begin{array}{l}\text { High saturated fatty acid } \\
\text { intake }\end{array}$ & $\begin{array}{l}\text { Low saturated fatty acid } \\
\text { intake }\end{array}$ & 2018 & $K=16$ & Ischemic & 11 & - & - & $0.88(0.81 ; 0.96)$ \\
\hline \multirow{5}{*}{ Narain [43] } & $\begin{array}{c}\text { High intake } \\
\text { sugar-sweetened beverages }\end{array}$ & $\begin{array}{c}\text { Low intake } \\
\text { sugar-sweetened } \\
\text { beverages }\end{array}$ & \multirow{5}{*}{2016} & \multirow{5}{*}{$\mathrm{K}=7$} & $\begin{array}{l}\text { Ischemic or } \\
\text { Hemorrhagic }\end{array}$ & 3 & 236061 & - & $1.10(0.97 ; 1.25)$ \\
\hline & $\begin{array}{c}\text { High intake } \\
\text { sugar-sweetened beverages }\end{array}$ & $\begin{array}{c}\text { Low intake } \\
\text { sugar-sweetened } \\
\text { beverages }\end{array}$ & & & $\begin{array}{l}\text { Ischemic stroke } \\
\text { (in men) }\end{array}$ & 3 & - & - & $1.01(0.74 ; 1.37)$ \\
\hline & $\begin{array}{c}\text { High intake } \\
\text { sugar-sweetened beverages }\end{array}$ & $\begin{array}{c}\text { Low intake } \\
\text { sugar-sweetened } \\
\text { beverages }\end{array}$ & & & $\begin{array}{l}\text { Ischemic stroke } \\
\text { (in women) }\end{array}$ & 3 & - & - & $1.33(1.07 ; 1.66)$ \\
\hline & $\begin{array}{l}\text { High intake } \\
\text { sugar-sweetened beverages }\end{array}$ & $\begin{array}{c}\text { Low intake } \\
\text { sugar-sweetened } \\
\text { beverages }\end{array}$ & & & $\begin{array}{l}\text { Hemorrhagic stroke } \\
\quad \text { (in men) }\end{array}$ & 3 & - & - & $0.87(0.68 ; 1.12)$ \\
\hline & $\begin{array}{c}\text { High intake } \\
\text { sugar-sweetened beverages }\end{array}$ & $\begin{array}{c}\text { Low intake } \\
\text { sugar-sweetened } \\
\text { beverages }\end{array}$ & & & $\begin{array}{l}\text { Hemorrhagic stroke } \\
\text { (in women) }\end{array}$ & 3 & - & - & $0.83(0.62 ; 1.10)$ \\
\hline \multirow{3}{*}{ Cai [44] } & Glycemic index & \multirow{3}{*}{-} & \multirow{3}{*}{2014} & \multirow{3}{*}{$\mathrm{K}=7$} & \multirow{3}{*}{$\begin{array}{l}\text { Ischemic or } \\
\text { Hemorrhagic }\end{array}$} & \multirow{3}{*}{7} & \multirow{3}{*}{-} & \multirow{3}{*}{ - } & $1.10(0.99 ; 1.21)$ \\
\hline & Glycemic load & & & & & & & & $1.19(1.05 ; 1.36)$ \\
\hline & Carbohydrate intake & & & & & & & & $1.12(0.93 ; 1.35)$ \\
\hline Yan [45] & High soy consumption & Low soy consumption & 2016 & $\mathrm{~K}=11$ & $\begin{array}{l}\text { Ischemic or } \\
\text { Hemorrhagic }\end{array}$ & 11 & - & - & $0.82(0.68 ; 0.99)$ \\
\hline \multirow{3}{*}{ Zhang [46] } & \multirow{3}{*}{ High fiber intake } & \multirow{3}{*}{ Low fiber intake } & \multirow{3}{*}{2013} & \multirow{3}{*}{$\mathrm{K}=11$} & $\begin{array}{l}\text { Ischemic or } \\
\text { Hemorrhagic }\end{array}$ & 11 & 325,627 & - & $0.83(0.74 ; 0.93)$ \\
\hline & & & & & Ischemic & 8 & - & - & $0.83(0.74 ; 0.93)$ \\
\hline & & & & & Hemorrhagic & 5 & - & - & $0.87(0.74 ; 1.05)$ \\
\hline
\end{tabular}


Table 1. Cont

\begin{tabular}{|c|c|c|c|c|c|c|c|c|c|}
\hline \multirow{2}{*}{ Author } & \multirow{2}{*}{ Food or Beverage } & \multirow{2}{*}{ Control Group } & \multirow{2}{*}{$\begin{array}{c}\text { Literature } \\
\text { Search Update }\end{array}$} & \multirow{2}{*}{$\begin{array}{c}\text { Number of } \\
\text { Primary Studies }\end{array}$} & \multirow{2}{*}{ Type of Strokes * } & \multirow{2}{*}{$\begin{array}{l}\text { Number of Studies for } \\
\text { Evaluated Strokes }\end{array}$} & \multicolumn{2}{|c|}{ Population } & \multirow{2}{*}{$\begin{array}{l}\text { Effect Size } \\
95 \% \text { CI }\end{array}$} \\
\hline & & & & & & & No. Total & No. Events & \\
\hline \multirow{5}{*}{ Zhang [47] } & \multirow{3}{*}{ Protein intake } & \multirow[t]{3}{*}{ - } & \multirow{5}{*}{2016} & \multirow{5}{*}{$\mathrm{K}=12$} & $\begin{array}{l}\text { Ischemic or } \\
\text { Hemorrhagic }\end{array}$ & 12 & \multirow{5}{*}{ - } & \multirow{5}{*}{ - } & $0.98(0.89 ; 1.07)$ \\
\hline & & & & & Ischemic & 8 & & & $0.94(0.80 ; 1.10)$ \\
\hline & & & & & Hemorrhagic & 4 & & & $1.05(0.97 ; 1.14)$ \\
\hline & Animal protein & - & & & $\begin{array}{l}\text { Ischemic or } \\
\text { Hemorrhagic }\end{array}$ & 8 & & & $0.94(0.75 ; 1.17)$ \\
\hline & Vegetable protein & - & & & $\begin{array}{l}\text { Ischemic or } \\
\text { Hemorrhagic }\end{array}$ & 8 & & & $0.90(0.82 ; 0.99)$ \\
\hline \multirow{2}{*}{ Qin [48] } & Lean fish & Fatty fish & 2018 & $\mathrm{~K}=5$ & $\begin{array}{l}\text { Ischemic or } \\
\text { Hemorrhagic }\end{array}$ & 5 & - & - & $0.88(0.74 ; 1.04)$ \\
\hline & High lean fish intake & Low lean fish intake & 2018 & $\mathrm{~K}=5$ & $\begin{array}{l}\text { Ischemic or } \\
\text { Hemorrhagic }\end{array}$ & 5 & - & - & $0.81(0.67 ; 0.99)$ \\
\hline Xun [49] & High fish intake & Low fish intake & 2012 & $\mathrm{~K}=16$ & $\begin{array}{l}\text { Ischemic or } \\
\text { Hemorrhagic }\end{array}$ & 16 & - & - & $0.91(0.85 ; 0.98) *$ \\
\hline \multirow{4}{*}{$\operatorname{Kim}[50]$} & High total meat intake & Low total meat intake & \multirow{4}{*}{2016} & \multirow{4}{*}{$\mathrm{K}=7$} & \multirow{4}{*}{$\begin{array}{l}\text { Ischemic or } \\
\text { Hemorrhagic }\end{array}$} & 6 & - & - & $1.18(1.09 ; 1.28)$ \\
\hline & High red meat intake & Low red meat intake & & & & 7 & - & - & $1.11(1.03 ; 1.20)$ \\
\hline & $\begin{array}{l}\text { High processed meat } \\
\text { intake }\end{array}$ & $\begin{array}{l}\text { Low processed meat } \\
\text { intake }\end{array}$ & & & & 8 & - & - & $1.17(1.08 ; 1.25)$ \\
\hline & High white meat intake & Low white meat intake & & & & 4 & - & - & $0.87(0.78 ; 0.96)$ \\
\hline Yuan [51] & High chocolate intake & Low chocolate intake & 2017 & $\mathrm{~K}=8$ & $\begin{array}{l}\text { Ischemic or } \\
\text { Hemorrhagic }\end{array}$ & 8 & - & - & $0.84(0.78 ; 0.90)$ \\
\hline Tang [52] & High flavonoids intake & Low flavonoids intake & 2016 & $\mathrm{~K}=11$ & $\begin{array}{l}\text { Ischemic or } \\
\text { Hemorrhagic }\end{array}$ & 11 & - & & $0.89(0.82 ; 0.97)$ \\
\hline \multirow{3}{*}{ Chen [53] } & \multirow{3}{*}{ High vitamin $C$ intake } & \multirow{3}{*}{ Low vitamin $C$ intake } & \multirow{3}{*}{2011} & \multirow{3}{*}{$\mathrm{K}=11$} & $\begin{array}{l}\text { Ischemic or } \\
\text { Hemorrhagic }\end{array}$ & 11 & - & - & $0.81(0.74 ; 0.90)$ \\
\hline & & & & & Ischemic & 4 & & . & $0.77(0.64 ; 0.92)$ \\
\hline & & & & & Hemorrhagic & 2 & - & - & $1.07(0.38 ; 3.00)$ \\
\hline \multirow{2}{*}{ Afshin [54] } & \multirow{2}{*}{ Legumes $100 \mathrm{~g} /$ week } & \multirow{2}{*}{ No consumption } & \multirow{2}{*}{2014} & \multirow{2}{*}{$K=6$} & Ischemic & 3 & - & - & $1.07(0.77 ; 1.50)$ \\
\hline & & & & & Hemorrhagic & 4 & - & - & $1.23(0.91 ; 1.66)$ \\
\hline $\begin{array}{c}\text { Alexander } \\
{[55]}\end{array}$ & 1 egg/day & $<2$ eggs/week & 2016 & $\mathrm{~K}=7$ & $\begin{array}{l}\text { Ischemic or } \\
\text { Hemorrhagic }\end{array}$ & 7 & - & - & $0.88(0.81 ; 0.97)$ \\
\hline \multicolumn{10}{|c|}{ RCT } \\
\hline Bolland [34] & $\begin{array}{l}\text { High Ca from dairy } \\
\text { products }\end{array}$ & $\begin{array}{l}\text { Low Ca from dairy } \\
\text { products }\end{array}$ & 2011 & $K=8$ & $\begin{array}{l}\text { Ischemic or } \\
\text { Hemorrhagic }\end{array}$ & 5 & - & - & $0.69(0.60 ; 0.81)$ \\
\hline & $\begin{array}{l}\text { Calcium supplement } \\
500 \mathrm{mg} \text { and D vitamin }\end{array}$ & Placebo & & & $\begin{array}{l}\text { Ischemic or } \\
\text { Hemorrhagic }\end{array}$ & 3 & 20,090 & 477 & $1.20(1.00 ; 1.43)$ \\
\hline
\end{tabular}


Table 1. Cont.

\begin{tabular}{|c|c|c|c|c|c|c|c|c|c|}
\hline \multirow{2}{*}{ Author } & \multirow{2}{*}{ Food or Beverage } & \multirow{2}{*}{ Control Group } & \multirow{2}{*}{$\begin{array}{l}\text { Literature } \\
\text { Search Update }\end{array}$} & \multirow{2}{*}{$\begin{array}{c}\text { Number of } \\
\text { Primary Studies }\end{array}$} & \multirow{2}{*}{ Type of Strokes * } & \multirow{2}{*}{$\begin{array}{l}\text { Number of Studies for } \\
\text { Evaluated Strokes }\end{array}$} & \multicolumn{2}{|c|}{ Population } & \multirow{2}{*}{$\begin{array}{l}\text { Effect Size } \\
95 \% \text { CI }\end{array}$} \\
\hline & & & & & & & No. Total & No. Events & \\
\hline \multirow[b]{2}{*}{ Tian [27] } & $\begin{array}{l}\text { Intervention regimen folic } \\
\text { acid (FA) \#\# only }\end{array}$ & No supplementation & \multirow[b]{2}{*}{2017} & \multirow[b]{2}{*}{$\mathrm{K}=11$} & \multirow{2}{*}{$\begin{array}{l}\text { Ischemic or } \\
\text { Hemorrhagic }\end{array}$} & \multirow[b]{2}{*}{11} & 21295 & 657 & $0.79(0.68 ; 0.92)$ \\
\hline & $\begin{array}{c}\text { Intervention regimen FA + } \\
\text { vitamin B }\end{array}$ & No supplementation & & & & & 27486 & 1589 & $0.91(0.82 ; 1.00)$ \\
\hline Arab [26] & Tea 3 cups & Tea 1 cup & 2009 & $K=9$ & $\begin{array}{l}\text { Ischemic or } \\
\text { Hemorrhagic }\end{array}$ & 9 & - & - & $0.770 .71 ; 0.85$ \\
\hline \multirow{2}{*}{$\begin{array}{l}\text { Abdelhamid } \\
{[35]}\end{array}$} & $\begin{array}{l}\text { High long-chain omega-3 } \\
\text { polyunsaturated fatty acids } \\
\text { (PUFAs) intake }\end{array}$ & Low PUFAs intake & \multirow{2}{*}{2018} & \multirow{2}{*}{$\mathrm{K}=32$} & $\begin{array}{l}\text { Ischemic or } \\
\text { Hemorrhagic }\end{array}$ & 28 & 89,358 & 1818 & $1.06(0.96-1.16)$ \\
\hline & $\begin{array}{l}\text { High alpha linoleic acid } \\
\text { intake }\end{array}$ & $\begin{array}{l}\text { Low alpha linoleic acid } \\
\text { intake }\end{array}$ & & & $\begin{array}{l}\text { Ischemic or } \\
\text { Hemorrhagic }\end{array}$ & 4 & 19,327 & 51 & $1.15(0.66 ; 2.01)$ \\
\hline Hooper [36] & Low omega- 6 & High omega-6 intake & 2018 & $\mathrm{~K}=4$ & $\begin{array}{l}\text { Ischemic or } \\
\text { Hemorrhagic }\end{array}$ & 4 & 3730 & 54 & $1.36(0.45 ; 4.11)$ \\
\hline $\begin{array}{c}\text { Abdelhamid } \\
\text { [37] }\end{array}$ & $\begin{array}{c}\text { High polyunsaturated fatty } \\
\text { acid intake }\end{array}$ & $\begin{array}{l}\text { Low polyunsaturated } \\
\text { fatty acid intake }\end{array}$ & 2018 & $\mathrm{~K}=11$ & $\begin{array}{l}\text { Ischemic or } \\
\text { Hemorrhagic }\end{array}$ & 11 & 14,724 & 165 & $1.06(0.96 ; 1.96)$ \\
\hline Hooper [39] & $\begin{array}{c}\text { Low saturated fatty acid } \\
\text { diet }\end{array}$ & $\begin{array}{c}\text { Low saturated fatty acid } \\
\text { diet }\end{array}$ & 2015 & $\mathrm{~K}=8$ & $\begin{array}{l}\text { Ischemic or } \\
\text { Hemorrhagic }\end{array}$ & 8 & 50,952 & 1125 & $1.00(0.89 ; 1.12)$ \\
\hline \multirow{3}{*}{$\operatorname{Bin}[42]$} & \multirow{3}{*}{ Vitamin E } & \multirow{3}{*}{ - } & \multirow{3}{*}{2011} & \multirow{3}{*}{$K=13$} & $\begin{array}{l}\text { Ischemic or } \\
\text { Hemorrhagic }\end{array}$ & 13 & 166,282 & - & $1.01(0.96 ; 1.07)$ \\
\hline & & & & & Ischemic & - & - & - & $1.01(0.94 ; 1.09)$ \\
\hline & & & & & Hemorrhagic & - & - & - & $1.12(0.94 ; 1.33)$ \\
\hline
\end{tabular}

* Where not specified, stroke events is in both sexes. \# Dietary pattern: high intake of all kinds of red and/or processed meats, refined grains, sweets, desserts, high-fat dairy products, and high-fat gravy. ${ }^{* *}$ OR (odds ratio). \#\# Folic acid. 
Table 2. Distribution of primary studies included in meta-analyses considered according to geographic area and type of nutritional support.

\begin{tabular}{|c|c|c|c|c|c|c|c|c|c|c|c|c|c|c|}
\hline \multirow{2}{*}{$\begin{array}{c}\text { Total } \\
n=386\end{array}$} & \multicolumn{2}{|c|}{$\begin{array}{c}\text { Australia } \\
n=6(1.5 \%)\end{array}$} & \multicolumn{2}{|c|}{$\begin{array}{c}\text { Canada } \\
n=4(1 \%)\end{array}$} & \multicolumn{2}{|c|}{$\begin{array}{c}\text { China-Singapore-Korea } \\
n=22(5.7 \%)\end{array}$} & \multicolumn{2}{|c|}{$\begin{array}{c}\text { Europe } \\
n=162(42 \%)\end{array}$} & \multicolumn{2}{|c|}{$\begin{array}{c}\text { Japan } \\
n=60(15.5 \%)\end{array}$} & \multicolumn{2}{|c|}{$\begin{array}{c}\text { USA } \\
n=130(33.67 \%)\end{array}$} & \multicolumn{2}{|c|}{$\begin{array}{c}\text { Latin Americ } \\
n=1(0.25 \%)\end{array}$} \\
\hline & $n$ & $\%$ & $n$ & $\%$ & $n$ & $\%$ & $n$ & $\%$ & $n$ & $\%$ & $n$ & $\%$ & $n$ & $\%$ \\
\hline \multicolumn{15}{|l|}{ Eating habits } \\
\hline Healthy diet & & & & & 4 & 19 & 5 & 3.1 & 6 & 10 & 1 & 0.77 & & \\
\hline Carbohydrates & & & & & & & 4 & 2.4 & & & 6 & 4.6 & & \\
\hline \multicolumn{15}{|l|}{ Beverages } \\
\hline Alcohol & & & & & 2 & 9 & 10 & 6.2 & 7 & 11.6 & 7 & 5.3 & & \\
\hline Tea & 1 & 17 & & & 1 & 9 & 3 & 1.8 & 3 & 5 & 7 & 5.3 & & \\
\hline Soft drinks & & & & & 1 & 9 & 1 & 0.6 & 1 & 1.6 & 3 & 2.3 & & \\
\hline Nutrients & & & & & & & & 0 & & & & 0 & & \\
\hline Omega-3 & 1 & 17 & 1 & 0.15 & 1 & 4 & 4 & 2.5 & 3 & 5 & 4 & 3.0 & 1 & 100 \\
\hline Folic acid & & & 3 & 0.75 & 1 & 4 & 9 & 5.5 & 1 & 1.6 & 1 & 0.7 & & \\
\hline Monounsaturated fatty acids & 1 & 17 & & & & & 3 & 1.8 & 2 & 3.3 & 4 & 3.1 & & \\
\hline Polyunsaturated fatty acids & & & & & & & 3 & 1.8 & & & 2 & 1.5 & & \\
\hline Omega-6 & & & & & & & 2 & 1.2 & & & 1 & 0.7 & & \\
\hline Flavonoids & & & & & 1 & 4 & 6 & 3.7 & & & 4 & 3.0 & & \\
\hline Vitamin E & & & & & 1 & 4 & 11 & 6.8 & 3 & 5 & 6 & 4.6 & & \\
\hline Vitamin C & & & & & 1 & 4 & 6 & 3.7 & & & 3 & 2.3 & & \\
\hline Calcium/vitamin D & & & & & & & 2 & 1.2 & & & 1 & 0.7 & & \\
\hline Food & & & & & & & & & & & & 0 & & \\
\hline Dried fruits & 2 & 34 & & & 1 & 4 & 3 & 1.8 & & 0 & 2 & 1.5 & & \\
\hline Saturated fatty acids & & & & & & & 2 & 1.2 & 5 & 8.3 & 2 & 1.5 & & \\
\hline Butter & & & & & & & 3 & 1.8 & & 0 & 5 & 3.8 & & \\
\hline Meat & & & & & & & 4 & 2.5 & & 0 & 1 & 0.7 & & \\
\hline Cereals & & & & & 1 & 4 & 8 & 4.9 & 3 & 5 & 1 & 0.7 & & \\
\hline Chocolate & & & & & & & 5 & 3.1 & 1 & 1.6 & 4 & 3.1 & & \\
\hline Fibers & & & & & 1 & 14 & 3 & 1.8 & 2 & 3.3 & 14 & 10.7 & & \\
\hline Fruits and vegetables & & & & & 3 & & 22 & 13.6 & 7 & 11.6 & 11 & 8.5 & & \\
\hline Milk & & & & & & & 6 & 3.7 & 2 & 3.3 & 5 & 3.9 & & \\
\hline Milk and derivatives & & & & & & & 5 & 3.1 & 3 & 5 & 2 & 1.5 & & \\
\hline Legumes & & & & & & & 2 & 1.2 & 2 & 3.3 & 8 & 6.1 & & \\
\hline Olive oil & & & & & & & 2 & 1.2 & & 0 & & & & \\
\hline Fish & & & & & 1 & 4 & 12 & 7.4 & 3 & 5 & 6 & 4.6 & & \\
\hline Protein & & & & & 1 & 4 & 2 & 1.2 & 3 & 5 & 2 & 1.5 & & \\
\hline Soy & & & & & 1 & 4 & 2 & 1.2 & 2 & 3.3 & 5 & 3.8 & & \\
\hline Yogurt & & & & & & & 7 & 4.3 & & 0 & 13 & 10 & & \\
\hline Eggs & & & & & & & 5 & 3.1 & 1 & 1.6 & 2 & 1.5 & & \\
\hline
\end{tabular}




\section{Discussion}

Our review aims to carry out an overview of meta-analyses about the impact of nutrition in the prevention of ischemic/hemorrhagic stroke. Compared to a recent review [56] we wanted to underline some aspects: first, the geographical setting of conducting individual primary studies; second, the study design of the primary studies (observational RCTs); and third, methodological quality of meta-analyses. With respect to the first point, it is important to underline that all primary studies came from countries with high income levels. This evidence shows that many countries are not represented, consequently, different lifestyles, ethnic groups, and potentially harmful or virtuous eating habits are not reported. Moreover, different production standards, regulated by different national or international legislation, could influence the final summary of the data in evidence.

Omega-3 and omega- 6 integrators are the most studied, both in meta-analyses of observational studies and RCTs. Discrepancies emerge regarding long-chain omega-3 between the meta-analysis of Larsson [17] and that of Abdelhamid [35]; this difference is likely attributable to a greater sample size in Larsson's meta-analysis and to more recent publications.

Another highly studied integrator is vitamin C (in China-Singapore-Korea, Europe and the USA). Vitamin $C$ could have a neuroprotective action due to its antioxidant activity.

However, a Japanese population-based study noted that vitamin $C$ neuroprotection activity would be more effective in non-smokers than smokers, demonstrating that overall lifestyle is responsible for cardiovascular events [57].

Flavonoids act similar to vitamin C. Studies have been conducted in Europe, the USA and China-Singapore-Korea area (Figure 2). Flavonoids perform a neuroprotective action through a triple mechanism: reducing reactive oxygen species (ROS), reducing intracellular concentration of glutamate, and inducing the production of nitric oxide (NO) by activating the enzyme NO-synthase, a powerful vasodilator [58].

The role of some vitamins in relation to cardiovascular risk has also been studied. B vitamins, in particular folic acid, may be linked to the improvement of endothelial function, associated with the increase of 5-methyltetrahydrofolate reductase with the reduction of the circulating homocysteine [59]. Instead vitamin E could play a role in endothelial homeostasis in respect to local inflammation, lipid metabolism, and the stability of atherosclerotic plaques [60].

Comparing the geographical areas examined, the USA and Europe show particular attention to lifestyles. In fact, numerous studies have been conducted in these continents also in relation to alcohol consumption (Figure 2, Table 2). This data could be considered as an indicator of awareness with respect to food education policies and social habits which, however, appear to be very different between different nations, as in the case of Europe [61]. It is well known how the adoption of a healthy diet, with an adequate intake of carbohydrates, greatly reduces cardiovascular risk and obesity $[62,63]$. With respect to the consumption of soft drinks, it is noted that in Narain's meta-analysis there is an increased risk for ischemic stroke in women [43]. A recent work by Mullie et al. [64] showed that the daily consumption of soft drinks and similar drinks increases the risk of mortality from cerebrovascular diseases. Regarding tea consumption there are primary studies (Figure 2, Table S2). Tea as a drink originated in Asia and consumption is widespread worldwide. Among the other substances contained in tea (Camellia sinensis) the beneficial effects are attributed mostly to catechins. Catechins are molecules with a positive effect on endothelial function [64]. The benefit of this product for both Asians and Non-Asians was shown in a meta-analysis by Arab et al. [26]. There are many studies on cereals in a large part of the areas considered (Figure 2). It is important to underline that the consumption of fresh fruit, nuts, and legumes entails a potential risk reduction [19,21,45,54].

Their consumption is encouraged by all the most recent guidelines on cardiovascular prevention $[56,65,66]$ even though there are notable differences between geographical areas and social context $[21,66]$. As pointed out by Lake et al., climate change could also affect the accentuation of inequalities in access to food and healthy food, particularly in developing countries $[67,68]$. 
The results of the studies regarding red meat are controversial. Excessive consumption of red meat and specially processed meat, studied in only two geographical areas (Europe and the USA), show an increase in risk; while moderate consumption of red meat does not lead to an alteration of the lipid structure or a significant pressure rise [69]. Moreover, cardiovascular risk could be mitigated by the adequate consumption of fruit and vegetables [70,71].

Finally, it is important to underline that some widespread types of cancer, such as colorectal and breast cancer [69-71], have many risk factors in common with cardiovascular diseases.

Particular importance is the intestinal microbiome. Some studies suggest that dysbiosis may favor ischemic stroke. A study by Yin et al. showed that the bacterial flora of patients with stroke was rich in some opportunistic bacteria (Enterobacter, Megasphaera, Oscillibacter, and Desulfobivrio) compared to saprophytic flora (Bacterioides, Prevotella and Faecalibacterium) [72]. A work by Xia et al. showed a substantial difference in the microbiome between ischemic patients and control subjects [73].

A limitation of the present study is related to the design of the study of primary studies. In fact, the basal conditions and the possible comorbidities of the subjects enrolled in these studies are not known.

\section{Conclusions}

Most physicians and health professionals underestimate the importance of food and lifestyles, smoking, consumption of alcohol, and daily exercise as stroke risk factor. It is very important to underline nutrition in stroke prevention.

This review reveals that choosing foods with a more favorable nutritional profile may help reduce the risk of cardiovascular diseases and stroke in particular. These indications can be specifically addressed to those classes of the population with an increased risk of stroke, using a "tailored" preventive medicine for individuals based on genetic predisposition, presence of other risk factors or predisposing lifestyles.

Although far from identifying a "superfood" with nutraceutical properties that can guarantee absolute well-being or zero risk, it is clear that the choice of a balanced diet can reduce the risk of stroke, a disease with high social costs.

In the nineteenth century, Ludwig Feuerbach wrote "You are what you eat". The research carried out so far on nutrition confirms this brilliant statement. Governments should back public health policies and promote healthy lifestyles.

Supplementary Materials: The following are available online at http://www.mdpi.com/1660-4601/16/19/3582/s1, Table S1: Risk of Bias assessment according to AMSTAR-2 scale, Table S2: Summary of dose response analysis in studies considered.

Author Contributions: E.A.: guarantor of the article, study concept and design, literature search, data analysis, and manuscript writing. P.M.A.: literature search, data abstraction, participant manuscript writing. L.R.: literature search and graphic processing. R.P.: literature search. All authors have approved the final version of this manuscript.

Funding: This research received no external funding.

Conflicts of Interest: The authors declare no conflicts of interest.

\section{References}

1. Krishnamurthi, R.V.; Feigin, V.L.; Forouzanfar, M.H.; Mensha, G.A.; Connor, M.; Bennett, D.A.; Moran, A.E.; Sacco, R.L.; Anderson, L.M.; Truelsen, T.; et al. Global Burden of Diseases, Injuries, Risk Factors Study 2010 [GBD 2010]; GBD Stroke Experts Group.] Global and regional burden of first-ever ischaemic and haemorrhagic stroke during 1990-2010: Findings from the Global Burden of Disease Study 2010. Lancet Glob. Health 2013, 1, e259-e281. [CrossRef]

2. Olesen, J.; Gustavsson, A.; Svensson, M.; Wittchen, H.U.; Jönsso, B. CDBE 2010 study group European Brain Council. The economic cost of brain disorders in Europe. Eur. J. Neurol. 2012, 19, 155-162. [CrossRef] [PubMed] 
3. Demaerschalk, B.M.; Hwang, H.M.; Leung, G. US cost burden of ischemic stroke: A systematic literature review. Am. J. Manag. Care 2010, 16, 525-533. [PubMed]

4. Alwan, A. Global Status Report on Noncommunicable Diseases 2010; World Health Organization: Geneva, Switzerland, 2011.

5. Alexander, D.D.; Bylsma, L.C.; Vargas, A.J.; Cohen, S.S.; Doucette, A.; Mohamed, M.; Irvin, S.R.; Miller, P.E.; Watson, H.; Fryzek, J.P. Dairy consumption and CVD: A systematic review and meta-analysis. Br. J. Nutr. 2016, 11, 737-750. [CrossRef] [PubMed]

6. Briggs, M.A.; Petersen, K.S.; Kris-Etherton, P.M. Saturated Fatty Acids and Cardiovascular Disease: Replacements for Saturated Fat to Reduce Cardiovascular Risk. Healthcare 2017, 5, e29. [CrossRef]

7. Zhang, X.; Shu, L.; Si, C.; Yu, X.; Gao, W.; Liao, D.; Zhang, L.; Liu, X.; Zheng, P. Dietary Patterns and Risk of Stroke in Adults: A Systematic Review and Meta-analysis of Prospective Cohort Studies. J. Stroke Cerebrovasc. Dis. 2015, 24, 2173-2182. [CrossRef]

8. Mullie, P.; Pizot, C.; Autier, P. Daily milk consumption and all-cause mortality, coronary heart disease and stroke: A systematic review and meta-analysis of observational cohort studies. BMC Public Health 2016, 16, 1236. [CrossRef]

9. Pimpin, L.; Wu, J.H.; Haskelberg, H.; Del Gobbo, L.; Mozaffarian, D. Is Butter Back? A systematic review and meta-analysis of butter consumption and risk of cardiovascular disease, diabetes, and total mortality. PLoS ONE 2016, 11, e0158118. [CrossRef]

10. Michaëlsson, K.; Wolk, A.; Langenskiold, S.; Basu, S.; Warensjo Lemming, E.; Melhus, H.; Byberg, L. Milk intake and risk of mortality and fractures in women and men: Cohort studies. BMJ 2014, 349, g6015. [CrossRef]

11. Soedamah-Muthu, S.S.; Ding, E.L.; Al-Delaimy, W.K.; Hu, F.B.; Engberink, M.F.; Willett, W.C.; Geleijnse, J.M. Milk and dairy consumption and incidence of cardiovascular diseases and all-cause mortality: dose-response meta-analysis of prospective cohort studies. Am. J. Clin. Nutr. 2011, 93, 158-171. [CrossRef]

12. Larsson, S.C.; Wallin, A.; Wolk, A.; Markus, H.S. Differing association of alcohol consumption with different stroke types: A systematic review and meta-analysis. BMC Med. 2016, 14, 178. [CrossRef] [PubMed]

13. Fernández-Solà, J. Cardiovascular risks and benefits of moderate and heavy alcohol consumption. Nat. Rev. Cardiol. 2015, 12, 576-587. [CrossRef] [PubMed]

14. Stephan, L.S.; Almeida, E.D.; Markoski, M.M.; Garavaglia, J.; Marcadenti, A. Red Wine, Resveratrol and Atrial Fibrillation. Nutrients 2017, 30, 9. [CrossRef] [PubMed]

15. De Gaetano, G.; Costanzo, S.; Di Castelnuovo, A.; Badimon, L.; Bejko, D.; Alkerwi, A.; Chiva-Blanch, G.; Estruch, R.; la Vecchia, C.; Panico, S.; et al. Effects of moderate beer consumption on health and disease: A consensus document. Nutr. Metab. Cardiovasc. Dis. 2016, 26, 443-467. [CrossRef] [PubMed]

16. Cheng, P.; Wang, J.; Shao, W. Monounsaturated fatty acid intake and stroke risk: A meta-analysis of prospective cohort studies. J. Stroke Cerebrovasc. Dis. 2016, 25, 1326-1334. [CrossRef] [PubMed]

17. Larsson, S.C.; Orsini, N.; Wolk, A. Long-chain omega-3 polyunsaturated fatty acids and risk of stroke: A meta-analysis. Eur. J. Epidemiol. 2012, 27, 895-901. [CrossRef] [PubMed]

18. Kris-Etherton, P.M.; Pearson, T.A.; Wan, Y.; Hargrove, R.L.; Moriarty, K.; Fishell, V.; Etherton, T.D. High-monounsaturated fatty acid diets lower both plasma cholesterol and triacylglycerol concentrations. Am. J. Clin. Nutr. 1999, 70, 1009-1015. [CrossRef] [PubMed]

19. Aune, D.; Giovannucci, E.; Boffetta, P.; Fadnes, L.T.; Keum, N.; Norat, T.; Greenwood, D.C.; Riboli, E.; Vatten, L.J.; Tonstad, S. Fruit and vegetable intake and the risk of cardiovascular disease, total cancer and all-cause mortality-A systematic review and dose-response meta-analysis of prospective studies. Int. J. Epidemiol. 2017, 46, 1029-1056. [CrossRef]

20. Lee-Kwan, S.E.; Moore, L.V.; Blanck, H.M.; Harris, D.M.; Galuska, D. Disparities in State-Specific Adult Fruit and Vegetable Consumption-United States 2015. MMWR Morb. Mortal. Wkly. Rep. 2017, 66, 1241-1247. [CrossRef]

21. Chen, G.C.; Zhang, R.; Martínez-González, M.A.; Zhang, Z.L.; Bonaccio, M.; van Dam, R.M.; Qin, L.Q. Nut consumption in relation to all-cause and cause-specific mortality: A meta-analysis 18 prospective studies. Food Funct. 2017, 8, 3893-3905. [CrossRef] 
22. Aune, D.; Keum, N.; Giovannucci, E.; Fadnes, L.T.; Boffetta, P.; Greenwood, D.C.; Tonstad, S.; Vatten, L.J.; Riboli, E.; Norat, T. Nut consumption and risk of cardiovascular disease, total cancer, all-cause and cause-specific mortality: A systematic review and dose-response meta-analysis of prospective studies. BMC Med. 2016, 14, 207. [CrossRef] [PubMed]

23. Del Gobbo, L.C.; Falk, M.C.; Feldman, R.; Lewis, K.; Mozaffarian, D. Effects of tree nuts on blood lipids, apolipoproteins, and blood pressure: Systematic review, meta-analysis, and dose-response of 61 controlled intervention trials. Am. J. Clin. Nutr. 2015, 102, 1347-1356. [CrossRef]

24. Pereira, M.A.; Kartashov, A.I.; Ebbeling, C.B.; Van Horn, L.; Slattery, M.L.; Jacobs, D.R., Jr.; Ludwig, D.S. Fast-food habits, weight gain, and insulin resistance [the CARDIA study]: 15-year prospective analysis. Lancet. 2005, 365, 36-42. [CrossRef]

25. Nomura, K.; Yamanouchi, T. The role of fructose-enriched diets in mechanisms of nonalcoholic fatty liver disease. J. Nutr. Biochem. 2012, 23, 203-208. [CrossRef] [PubMed]

26. Arab, L.; Li, U.W.; Elashoff, D. Green and black tea consumption and risk of stroke: A meta-analysis. Stroke 2009, 40, 1786-1792. [CrossRef]

27. Tian, T.; Yang, K.Q.; Cui, J.G.; Zhou, L.L.; Zhou, X.L. Folic Acid Supplementation for Stroke Prevention in Patients with Cardiovascular Disease. Am. J. Med. Sci. 2017, 354, 379-387. [CrossRef] [PubMed]

28. Yang, H.T.; Lee, M.; Hong, K.S.; Ovbiagele, B.; Saver, J.L. Efficacy of folic acid supplementation in cardiovascular disease prevention: An updated meta-analysis of randomized controlled trials. Eur. J. Intern. Med. 2012, 23, 745-754. [CrossRef] [PubMed]

29. Miller, E.R., 3rd; Juraschek, S.; Pastor-Barriuso, R.; Bazzano, L.A.; Appel, L.J.; Guallar, E. Meta-analysis of folic acid supplementation trials on risk of cardiovascular disease and risk interaction with baseline homocysteine levels. Am. J. Cardiol. 2010, 106, 517-527. [CrossRef]

30. Aune, D.; Keum, N.; Giovannucci, E.; Fadnes, L.T.; Boffetta, P.; Greenwood, D.C.; Tonstad, S.; Vatten, L.J.; Riboli, E.; Norat, T. Whole grain consumption and risk of cardiovascular disease, cancer, and all cause and cause specific mortality: Systematic review and dose-response analysis of prospective studies. BMJ 2016, 353, i2716. [CrossRef]

31. Moher, D.; Liberati, A.; Tetzlaff, J.; Altman, D.G.; PRISMA Group. Preferred reporting items for systematic reviews and meta-analyses: The PRISMA statement. J. Clin. Epidemiol. 2009, 62, 1006-1012. [CrossRef]

32. Shea, B.J.; Reeves, B.C.; Wells Thuku, M.; Hamel, C.; Moran, J.; Moher, D.; Tugwell, P.; Welch, V.; Kristjansson, E.; Henry, D.A. AMSTAR 2: A critical appraisal tool for systematic reviews that include randomised or non-randomised studies of healthcare interventions, or both. BMJ 2017, 358, j4008. [CrossRef] [PubMed]

33. Wu, L.; Sun, D. Consumption of Yogurt and the Incident Risk of Cardiovascular Disease: A Meta-Analysis of Nine Cohort Studies. Nutrients 2017, 9, 315. [CrossRef] [PubMed]

34. Bolland, M.J.; Grey, A.; Avenell, A.; Gamble, G.D.; Reid, I.R. Calcium supplements with or without vitamin $\mathrm{D}$ and risk of cardiovascular events: Reanalysis of the Women's Health Initiative limited access dataset and meta-analysis. BMJ 2011, 342, d2040. [CrossRef] [PubMed]

35. Abdelhamid, A.S.; Brown, T.J.; Brainard, J.S.; Biswas, P.; Thorpe, G.C.; Moore, H.J.; Deane, K.H.; AlAbdulghafoor, F.K.; Summerbell, C.D.; Worthington, H.V.; et al. Omega-3 fatty acids for the primary and secondary prevention of cardiovascular disease. Cochrane Database Syst. Rev. 2018, 11, CD003177. [PubMed]

36. Hooper, L.; Al-Khudairy, L.; Abdelhamid, A.S.; Rees, K.; Brainard, J.S.; Brown, T.J.; Ajabnoor, S.M.; O'Brien, A.T.; Winstanley, L.E.; Donaldson, D.H.; et al. Omega-6 fats for the primary and secondary prevention of cardiovascular disease. Cochrane Database Syst. Rev. 2018, 11, CD011094. [PubMed]

37. Abdelhamid, A.S.; Martin, N.; Bridges, C.; Brainard, J.S.; Wang, X.; Brown, T.J.; Hanson, S.; Jimoh, O.F.; Ajabnoor, S.M.; Deane, K.H.; et al. Polyunsaturated fatty acids for the primary and secondary prevention of cardiovascular disease. Cochrane Database Syst. Rev. 2018, 11, CD012345.

38. Muto, M.; Ezaki, O. High Dietary Saturated Fat is Associated with a Low Risk of Intracerebral Hemorrhage and Ischemic Stroke in Japanese but not in Non-Japanese: A Review and Meta-Analysis of Prospective Cohort Studies. J. Atheroscler. Thromb. 2018, 25, 375-392. [CrossRef] [PubMed]

39. Hooper, L.; Martin, N.; Abdelhamid, A.; Davey Smith, G. Reduction in saturated fat intake for cardiovascular disease. Cochrane Database Syst. Rev. 2015, 6, CD01173. [CrossRef] 
40. Martínez-González, M.A.; Dominguez, L.J.; Delgado-Rodríguez, M. Olive oil consumption and risk of CHD and/or stroke: A meta-analysis of case-control, cohort and intervention studies. Br. J. Nutr. 2014, 112, 248-259. [CrossRef]

41. Bin, Q.; Hu, X.; Cao, Y.; Gao, F. The role of vitamin E (tocopherol) supplementation in the prevention of stroke. A meta-analysis of 13 randomised controlled trials. Thromb. Haemost. 2011, 105, 579-585. [CrossRef]

42. Cheng, P.; Wang, L.; Ning, S.; Liu, Z.; Lin, H.; Chen, S.; Zhu, J. Vitamin E intake and risk of stroke: A meta-analysis. Br. J. Nutr. 2018, 120, 1181-1188. [CrossRef] [PubMed]

43. Narain, A.; Kwok, C.S.; Mamas, M.A. Soft drinks and sweetened beverages and the risk of cardiovascular disease and mortality: A systematic review and meta-analysis. Int. J. Clin. Pract. 2016, 70, 791-805. [CrossRef] [PubMed]

44. Cai, X.; Wang, C.; Wang, S.; Cao, G.; Jin, C.; Yu, J.; Li, X.; Yan, J.; Wang, F.; Yu, W.; et al. Carbohydrate Intake, Glycemic Index, Glycemic Load, and Stroke: A Meta-analysis of Prospective Cohort Studies. Asia Pac. J. Public Health 2015, 27, 486-496. [CrossRef] [PubMed]

45. Yan, Z.; Zhang, X.; Li, C.; Jiao, S.; Dong, W. Association between consumption of soy and risk of cardiovascular disease: A meta-analysis of observational studies. Eur. J. Prev. Cardiol. 2017, 24, 735-747. [CrossRef] [PubMed]

46. Zhang, Z.; Xu, G.; Liu, D.; Zhu, W.; Fan, X.; Liu, X. Dietary fiber consumption and risk of stroke. Eur. J. Epidemiol. 2013, 28, 119-130. [CrossRef] [PubMed]

47. Zhang, X.W.; Yang, Z.; Li, M.; Li, K.; Deng, Y.Q.; Tang, Z.Y. Association between dietary protein intake and risk of stroke: A meta-analysis of prospective studies. Int. J. Cardiol. 2016, 223, 548-551. [CrossRef] [PubMed]

48. Qin, Z.Z.; Xu, J.Y.; Chen, G.C.; Ma, Y.X.; Qin, L.Q. Effects of fatty and lean fish intake on stroke risk: A meta-analysis of prospective cohort studies. Lipids Health Dis. 2018, 17, 264. [CrossRef]

49. Xun, P.; Qin, B.; Song, Y.; Nakamura, Y.; Kurth, T.; Yaemsiri, S.; Djousse, L.; He, K. Fish consumption and risk of stroke and its subtypes: Accumulative evidence from a meta-analysis of prospective cohort studies. Eur. J. Clin. Nutr. 2012, 66, 1199-1207. [CrossRef]

50. Kim, K.; Hyeon, J.; Lee, S.A.; Kwon, S.O.; Lee, H.; Keum, N.; Lee, J.K.; Park, S.M. Role of Total, Red, Processed, and White Meat Consumption in Stroke Incidence and Mortality: A Systematic Review and Meta-Analysis of Prospective Cohort Studies. J. Am. Heart Assoc. 2017, 6, e005983. [CrossRef]

51. Yuan, S.; Li, X.; Jin, Y.; Lu, J. Chocolate Consumption and Risk of Coronary Heart Disease, Stroke, and Diabetes: A Meta-Analysis of Prospective Studies. Nutrients 2017, 9, e688. [CrossRef]

52. Tang, Z.; Li, M.; Zhang, X.; Hou, W. Dietary flavonoid intake and the risk of stroke: A dose-response meta-analysis of prospective cohort studies. BMJ Open 2016, 6, e008680. [CrossRef] [PubMed]

53. Chen, G.C.; Lu, D.B.; Pang, Z.; Liu, Q.F. Vitamin C intake, circulating vitamin C and risk of stroke: A meta-analysis of prospective studies. J. Am. Heart Assoc. 2013, 2, e000329. [CrossRef] [PubMed]

54. Afshin, A.; Micha, R.; Khatibzadeh, S.; Mozaffarian, D. Consumption of nuts and legumes and risk of incident ischemic heart disease, stroke, and diabetes: A systematic review and meta-analysis. Am. J. Clin. Nutr. 2014, 100, 278-288. [CrossRef] [PubMed]

55. Alexander, D.D.; Miller, P.E.; Vargas, A.J.; Weed, D.L.; Cohen, S.S. Meta-analysis of Egg Consumption and Risk of Coronary Heart Disease and Stroke. J. Am. Coll. Nutr. 2016, 35, 704-716. [CrossRef] [PubMed]

56. Iacoviello, L.; Bonaccio, M.; Cairella, G.; Catani, M.V.; Costanzo, S.; D’Elia, L.; Giacco, R.; Rendina, D.; Sabino, P.; Savini, I.; et al. Diet and primary prevention of stroke: Systematic review and dietary recommendations by the ad hoc Working Group of the Italian Society of Human Nutrition. Nutr. Metab. Cardiovasc. Dis. 2018, 28, 309-334. [CrossRef] [PubMed]

57. Uesugi, S.; Ishihara, J.; Iso, H.; Sawada, N.; Takachi, R.; Inoue, M.; Tsugane, S. Dietary intake of antioxidant vitamins and risk of stroke: The Japan Public Health Center-based Prospective Study. Eur. J. Clin. Nutr. 2017, 71, 1179-1185. [CrossRef]

58. Tressera-Rimbau, A.; Arranz, S.; Eder, M.; Vallverdú-Queralt, A. Dietary Polyphenols in the Prevention of Stroke. Oxid. Med. Cell. Longev. 2017, 2017, 7467962. [CrossRef]

59. Shirodaria, C.; Antoniades, C.; Lee, J.; Jackson, C.E.; Robson, M.D.; Francis, J.M.; Moat, S.J.; Ratnatunga, C.; Pillai, R.; Refsum, H.; et al. Global improvement of vascular function and redox state with low-dose folic acid: Implications for folate therapy in patients with coronary artery disease. Circulation 2007, 115, 2262-2270. [CrossRef] [PubMed] 
60. Sozen, E.; Demirel, T.; Ozer, N.K. Vitamin E: Regulatory Role in the cardiovascular system. IUBMB Life 2019, 71, 507-515. [CrossRef]

61. Altobelli, E.; Rapacchietta, L.; Angeletti, P.M.; Barbante, L.; Profeta, F.V.; Fagnano, R. Breast cancer screening programmes across the who european region: Differences among countries based on national income level. Int. J. Environ. Res. Public Health 2017, 4, e452. [CrossRef]

62. Altobelli, E.; Rapacchietta, L.; Marziliano, C.; Campagna, G.; Profeta, V.F.; Fagnano, R. Differences in colorectal cancer surveillance epidemiology and screening in the WHO European Region. Oncol. Lett. 2019, 17, 2531-2542. [CrossRef] [PubMed]

63. Hu, T.; Bazzano, L.A. The low-carbohydrate diet and cardiovascular risk factors: Evidence from epidemiologic studies. Nutr. Metab. Cardiovasc. Dis. 2014, 24, 337-343. [CrossRef] [PubMed]

64. Mullee, A.; Romaguera, D.; Pearson-Stuttard, J.; Viallon, V.; Stepien, M.; Freisling, H.; Fagherazzi, G.; Mancini, F.R.; Boutron-Ruault, M.C.; Kühn, T.; et al. Association Between Soft Drink Consumption and Mortality in 10 European Countries. JAMA Intern. Med. 2019. [CrossRef] [PubMed]

65. Jochmann, N.; Lorenz, M.; Krosigk, A.V.; Martus, P.; Böhm, V.; Baumann, G.; Stangl, K.; Stangl, V. The efficacy of black tea in ameliorating endothelial function is equivalent to that of green tea. Br. J. Nutr. 2008, 99, 863-868. [CrossRef]

66. Pandian, J.D.; Gall, S.L.; Kate, M.P.; Silva, G.S.; Akinyemi, R.O.; Ovbiagele, B.I.; Lavados, P.M.; Gandhi, D.B.C.; Thrift, A.G. Prevention of stroke: A global perspective. Lancet 2018, 392, 1269-1278. [CrossRef]

67. Lake, I.R.; Hooper, L.; Abdelhamid, A.; Bentham, G.; Boxall, A.B.; Draper, A.; Fairweather-Tait, S.; Hulme, M.; Hunter, P.R.; Nichols, G.; et al. Climate change and food security: Health impacts in developed countries. Environ. Health Perspect. 2012, 120, 1520-1526. [CrossRef]

68. Ray, D.K.; West, P.C.; Clark, M.; Gerber, J.S.; Prishchepov, A.V.; Chatterjee, S. Climate change has likely already affected global food production. PLoS ONE 2019, 14, e0217148. [CrossRef]

69. Willett, W.C.; Stampfer, M.J. Current evidence on healthy eating. Annu. Rev. Public Health 2013, 34, 77-95. [CrossRef]

70. O'Connor, L.E.; Kim, J.E.; Campbell, W.W. Total red meat intake of $\geq 0.5$ servings/day does not negatively influence cardiovascular disease risk factors: A systemically searched meta-analysis of randomized controlled trials. Am. J. Clin. Nutr. 2017, 105, 57-69. [CrossRef]

71. Bellavia, A.; Stilling, F.; Wolk, A. High red meat intake and all-cause cardiovascular and cancer mortality: Is the risk modified by fruit and vegetable intake? Am. J. Clin. Nutr. 2016, 104, 1137-1143. [CrossRef]

72. Yin, J.; Liao, S.X.; He, Y.; Wang, S.; Xia, G.H.; Liu, F.T.; Zhu, J.J.; You, C.; Chen, Q.; Zhou, L.; et al. Dysbiosis of Gut Microbiota with Reduced Trimethylamine-N-Oxide Level in Patients with Large-Artery Atherosclerotic Stroke or Transient Ischemic Attack. J. Am. Heart Assoc. 2015, 4, e02699. [CrossRef] [PubMed]

73. Xia, G.H.; You, C.; Gao, X.X.; Zeng, X.L.; Zhu, J.J.; Xu, K.Y.; Tan, C.H.; Xu, R.T.; Wu, Q.H.; Zhou, H.W.; et al. Stroke Dysbiosis Index (SDI) in Gut Microbiome Are Associated with Brain Injury and Prognosis of Stroke. Front. Neurol. 2019, 10, 397. [CrossRef] [PubMed]

(C) 2019 by the authors. Licensee MDPI, Basel, Switzerland. This article is an open access article distributed under the terms and conditions of the Creative Commons Attribution (CC BY) license (http://creativecommons.org/licenses/by/4.0/). 